\title{
Convergence of a Random Walk Method for the Burgers Equation
}

\author{
By Stephen Roberts
}

Dedicated to Professor Eugene Isaacson on the occasion of his 70th birthday

\begin{abstract}
We show that the solution of the Burgers equation can be approximated in $L^{1}(\mathbf{R})$, to within $O\left(m^{-1 / 4}(\ln m)^{2}\right)$, by a random walk method generated by $O(m)$ particles. The nonlinear advection term of the equation is approximated by advecting the particles in a velocity field induced by the particles. The diffusive term is approximated by adding an appropriate random perturbation to the particle positions. It is also shown that the corresponding viscous splitting algorithm approximates the solution of the Burgers equation in $L^{1}(\mathbf{R})$ to within $O(k)$ when $k$ is the size of the time step. This work provides the first proof of convergence in a strong sense, for a random walk method, in which the related advection equation allows for the formation of shocks.
\end{abstract}

1. Introduction. In this paper we will prove the convergence of a numerical method used to solve the Burgers equation

$$
\partial_{t} u+u \partial_{x} u=\nu \partial_{x}^{2} u, \quad u(x, 0)=u_{0}(x),
$$

which incorporates a random walk technique to approximate the diffusion component of the equation. This result first appeared in Roberts [31] and was the first proof of convergence of a random walk method, in a strong sense ( $L^{p}$-norm, some $p$ ), in which the associated advection equation (in this case the inviscid Burgers equation) allows for the formation of shocks.

The Burgers equation was advanced by Burgers [4] as a one-dimensional model for the Navier-Stokes equations. In a similar manner the numerical method that we present is to be considered as a model for the random vortex method (Chorin [6]), a method which has been used extensively to solve the incompressible Navier-Stokes equations.

Our numerical method is a fractional step method (see Richtmyer and Morton [30, §8.9], Chorin et al. [10], and Chernoff [5] for a discussion of fractional step methods).

The first step of our method approximates the solution of the inviscid Burgers equation

$$
\partial_{t} u+u \partial_{x} u=0, \quad u(x, 0)=u_{0}(x)
$$

We suppose that the gradient of the solution is approximated by a collection of particles, so that

$$
\partial_{x} u(x, t) \simeq \sum_{i=1}^{m} \delta\left(x-x_{i}(t)\right) w_{i},
$$

Received December 1, 1986; revised October 23, 1987.

1980 Mathematics Subject Classification (1985 Revision). Primary 65M15, 65U05. 
where $x_{i}(t)$ signifies the position of each particle at time $t, w_{i}$ denotes the strength of each particle and $\delta(x)$ denotes the delta function concentrated at 0 . The approximate solution of (2) is obtained by allowing the positions of the particles to move with a velocity induced by the step function solution generated by the particles.

The second fractional step of our method involves solving the diffusion equation

$$
\partial_{t} u=\nu \partial_{x}^{2} u, \quad u(x, 0)=u_{0}(x)
$$

by utilizing the correspondence between the probability distribution of the position of a particle undergoing a random walk and the solution of the diffusion equation, as discussed in Einstein [13], Feller [15], Chorin and Marsden [9] and Chorin [6].

In essence, the diffusion is simulated by randomly perturbing the positions of the particles that generate the numerical solution. We notice that the statistical errors of our method are greatly reduced since our numerical solution is obtained by integrating the function generated by the particles. In random walk methods it is advantageous to move particles which generate the gradient of the solution instead of particles which generate the solution itself.

The random vortex method, [6], is also a fractional step method; the first step involves advecting a collection of 'vortex particles' using an approximation of Euler's equations; the second step diffuses the particles as in our method. If boundaries are present, it is necessary to add an additional fractional step in which particles are created on the boundary to satisfy the boundary conditions. This method has proved to be a practical tool in the study of incompressible fluid flow; see for example Laitone [26], Stansby and Dixon [32], Sung et al. [33], Teng [34] and Van der Vegt and Huijsmans [35].

Similar 'random walk' methods have also been developed to solve other problems which contain diffusion (see Ghoniem and Sherman [18]). A random vortex sheet method has been developed to solve the Prandtl boundary layer equation (Chorin [7]). A combination of the random vortex method and the random vortex sheet method has been used to study turbulent combustion (see Ghoniem et al. [17] and Oppenheim and Ghoniem [29]). In addition, random walk methods have been developed for the solution of scalar reaction diffusion equations (see Brenier [2], [3], Chorin [8] and Hald [20]). In all of these methods, the diffusive part of the equation is solved by applying a random walk technique to a set of particle positions (Brenier [3] uses a pseudorandom walk technique).

The usefulness of these random walk methods depends on the following facts:

(1) If the Reynolds number for the equation is large ( $\nu$ small), then it may be computationally too expensive to use a standard finite difference scheme to solve the equation. Random walk methods produce little, if any numerical diffusion and so the computational labor for these methods is essentially independent of the Reynolds number.

(2) The analogy between a random walk method and the underlying physical process usually justifies the good qualitative behavior of these methods.

The convergence of these methods has still to be proved in a completely satisfactory sense. Marchioro and Pulvirenti [27] were the first to show that the random vortex method in two dimensions is convergent in a weak sense to the solution 
of the Navier-Stokes equation. From a numerical standpoint, weak convergence is unsatisfactory, one of the standard norms $\left(L^{1}, L^{\infty}\right)$ being preferred.

An important result has been reported by Goodman [19] in which he shows that the two-dimensional random vortex method does converge in the strong sense and in an appropriate probabilistic sense to the solution of the Navier-Stokes equation. His result depends on the work of Beale and Majda [1], in which the viscous splitting of the Navier-Stokes equation is shown to converge strongly, where the viscous splitting algorithm is the fractional step algorithm consisting of exactly solving the Euler equation and the diffusion equation.

Hald [21] has proved the strong convergence of a random walk method for a coupled system of diffusion equations with boundary. This is the first proof of convergence of a random walk method in which particles are created at the boundary to satisfy the boundary conditions. Hald [20] has also proved the strong convergence of a method for solving a reaction diffusion equation. Unfortunately, his method does not readily generalize to equations with advection. Brenier [3] has generalized Chorin's [8] reaction diffusion method to the case of scalar reaction advection diffusion equations. His method is very similar to our method for the Burgers equation, in that particles are moved via the action of the velocity field generated by the particles, and the diffusion is simulated by adding random perturbations to the particle positions. The approximation of the reaction step of his equation is undertaken by changing the strengths of the particles in an appropriate way. Brenier has been able to prove the $L^{1}$ convergence of a modified version of his method in which the diffusion algorithm is solved using a deterministic 'random walk' algorithm.

The purpose of this paper is to prove the strong convergence of our random walk method, in an appropriate probabilistic sense, and provide an estimate of the rate of convergence. To this end we will prove an analogous viscous splitting result for the Burgers equation as that obtained by Beale and Majda [1] for the Navier-Stokes equation. The probabilistic part of our proof uses a similar 'exponential bound' as used by Goodman [19], but the details of the proof are necessarily different. This stems from the fact that the velocity field given by our numerical algorithm for solving the inviscid Burgers equation does not depend continuously on the positions of the particles, whereas the numerical algorithm used in the random vortex method for solving the Euler equation does produce a velocity field which depends continuously on the particle positions. This derives from the fact that the solution of the inviscid Burgers equation can develop shocks, so that the velocity of a particle can change dramatically depending on which side of a shock it is placed. On the other hand, the solution of the two-dimensional Euler's equation is smooth for all time, given smooth initial data, McGrath [28].

2. The Method. To describe our method, let us introduce some notation. The symbols $F_{t}, A_{t}$ and $D_{t}$ will denote the solution operators for equations (1), (2) and (3), respectively; i.e., $F_{t}$ is the solution operator for the full equation, i.e., Burgers equation, $A_{t}$ is the operator associated with advection, or equivalently, with the inviscid Burgers equation, and $D_{t}$ is associated with diffusion.

We will denote the operators that approximate the advection and diffusion operators by $\tilde{A}_{t}$ and $\tilde{D}_{t}$, where we understand that these operators depend on a spatial 
parameter $h$, which denotes the absolute strength of the particles that generate the numerical solution.

The numerical approximation of the solution at time $n k\left(n \in \mathbf{Z}^{+}\right)$is obtained as follows.

Step 1: Initial Approximation. The initial step of the algorithm involves approximating the smooth initial data $u_{0}$ with a step function $S^{0} u_{0}$, which is generated by particles with position $X_{i}^{0}$ and strength $w_{i}, i=1, \ldots, m$, such that $\left|w_{i}\right|=h$. The initial step function approximation is given by

$$
S^{0} u_{0}(y)=u^{L}+\sum_{i=1}^{m} H\left(y-X_{i}^{0}\right) w_{i},
$$

where $u^{L}$ is the limit of $u_{0}$ at minus infinity and $H$ is the Heaviside function

$$
\begin{aligned}
H(y)=1 & \text { if } y>0 \\
\frac{1}{2} & \text { if } y=0 \\
0 & \text { if } y<0 .
\end{aligned}
$$

If we assume that $u_{0}$ is constant outside a compact set, then we may define

$$
S^{0} u_{0}(y)=h \llbracket u_{0} / h+1 / 2 \rrbracket \quad \text { almost everywhere, }
$$

where $\llbracket y \rrbracket$ denotes the largest integer less than or equal to $y$. Note that if $u_{0}$ is assumed to have bounded total variation, then $S^{0} u_{0}$ as defined will only have a finite number of discontinuities, corresponding to a finite number of particles.

Step 2: Approximate Advection. Given particles at position $X_{i}^{j}, i=1, \ldots, m$, at the end of the $j$ th time step, we need to evolve the particle positions in such a way that the associated step function approximates the solution of the inviscid Burgers equation.

We first observe that for small times, the weak entropy solution of the inviscid Burgers equation, with step function as initial data, can be obtained by splicing together the Riemann problem solutions associated with each discontinuity considered separately (see Whitham [36] for a discussion of the Riemann problem). The approximation operator is designed to move the particles along straight line paths that produce step functions which closely approximate the behavior of the appropriate Riemann problem solutions.

As time proceeds, the Riemann problem solutions generating the exact solutions start to interact and so produce a much more complicated solution. Similarly, the straight line trajectories of the particles will eventually intersect. At this time we consider the approximating step function as new initial data. We can then define new particle trajectories which approximate the exact solution with this new initial data, which for small time is given by a new collection of Riemann problem solutions. Hence we define the particle trajectories $x_{i}(t)$ with initial positions $x_{i}$ by the following inductive argurnent.

We let $t^{0}=0$ and $x_{i}(0)=x_{i}$. Suppose that at a time $t^{k}$ we have $x_{i}(t)$ defined for $0 \leq t \leq t^{k}$. Let us define

$$
S(y, t)=u^{L}+\sum_{i=1}^{m} H\left(y-x_{i}(t)\right) w_{i}
$$


for $0 \leq t \leq t^{k}$ and denote the position of the particles at time $t^{k}$ by $x_{i}^{k}$. The strength of the discontinuity at the $i$ th particle position of the step function generated by the particles at time $t^{k}$ is

$$
[S]_{i}^{k}=S\left(x_{i}^{k}+0, t^{k}\right)-S\left(x_{i}^{k}-0, t^{k}\right) .
$$

It is easy to see that $[S]_{i}^{k}$ is the total strength of all the particles positioned at $x_{i}^{k}$ at time $t^{k}$.

The solution of the Riemann problem for the inviscid Burgers equation takes two distinct forms depending on the sign of the discontinuity, one being a shock solution if $[S]_{i}^{k}<0$, the other being a rarefaction wave if $[S]_{i}^{k}>0$ (see Whitham $[36]$ ). Hence the way in which the $i$ th particle is transported for time $t>t^{k}$ will be determined by the sign of the quantity $[S]_{i}^{k}$.

For the particles with $[S]_{i}^{k} \leq 0$, we let the trajectories of the particles coincide with the shock that occurs in the exact solution. This is accomplished by moving all of the particles that generate a specific negative discontinuity with a common velocity given by the Rankine-Hugoniot condition for that discontinuity (see Figures $1 \mathrm{a}, \mathrm{b})$.

Hence we define the velocity $S_{i}^{k}$ of the $i$ th particle at time $t^{k}$ to be

$$
S_{i}^{k}=\frac{1}{2}\left[S\left(x_{i}^{k}+0, t^{k}\right)+S\left(x_{i}^{k}-0, t^{k}\right)\right] .
$$

On the other hand, if $[S]_{i}^{k}>0$ we define the velocity of the particles generating this positive discontinuity in such a way as to approximate the exact rarefaction wave solution. Suppose the discontinuity at time $t^{k}$ is generated by $q$ particles with positive strength and $p$ particles with negative strength. If the $i$ th particle is one of the first $q-p$ particles with positive strength, then we define the particle velocity as

$$
S_{i}^{k}=S\left(x_{i}^{k}-0, t^{k}\right)+\sum_{\substack{j<i, x_{j}=x_{i} \\ w,>0}} w_{j}+\frac{1}{2} w_{i}
$$

(see Figure 1). All of the other $2 p$ particles, namely the particles with negative strength and the remaining particles with positive sign are given a common velocity

$$
S_{i}^{k}=S\left(x_{i}^{k}+0, t^{k}\right) .
$$

Note that $S_{i}^{k}$ depends both on the ordering of the particles relative to the index $i$ and on the ordering of the particle positions.

Let

$$
t^{k+1}=\min _{i, j}\left\{t^{k}+\frac{x_{j}^{k}-x_{i}^{k}}{S_{i}^{k}-S_{j}^{k}}: x_{j}^{k}>x_{i}^{k} \text { and } S_{i}^{k}>S_{j}^{k}\right\}
$$

(if no such $i, j$ exist then $t^{k+1}=\infty$ ). The position of the particles for times $t^{k} \leq t \leq t^{k+1}$ are given by the equation

$$
x_{i}(t)=x_{i}^{k}+\left(t-t^{k}\right) S_{i}^{k} .
$$

Notice that time $t^{k+1}$ gives the first time of intersection, after time $t^{k}$, of the particle trajectories of at least two particles that were at different positions at time $t^{k}$ (see Figure 1b). 


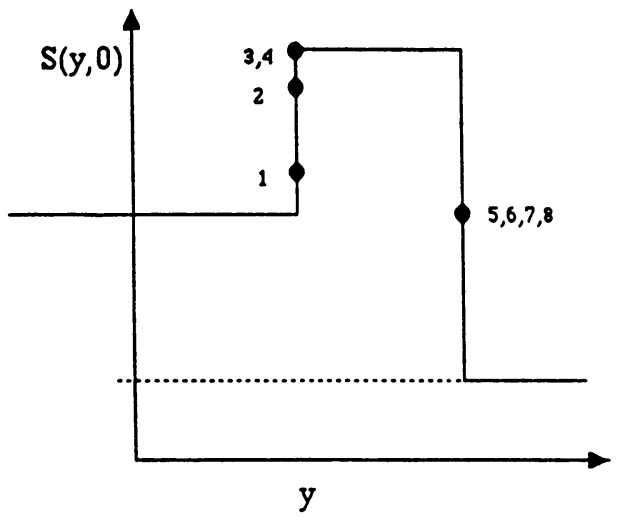

(a) Step function generated at time $t^{0}$.

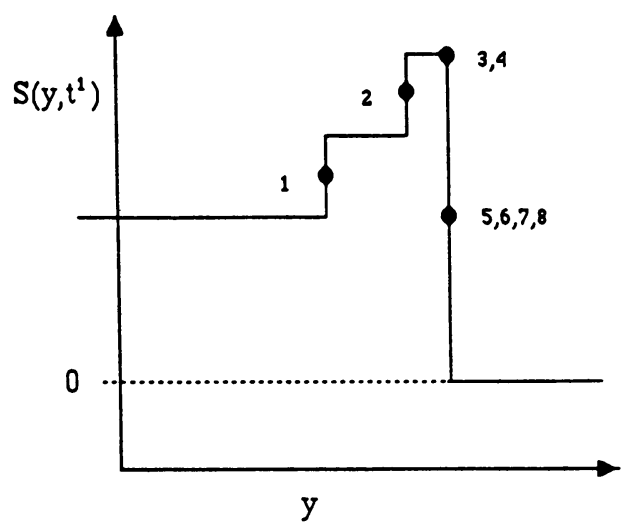

(c) Approximation at time $t^{1}$.

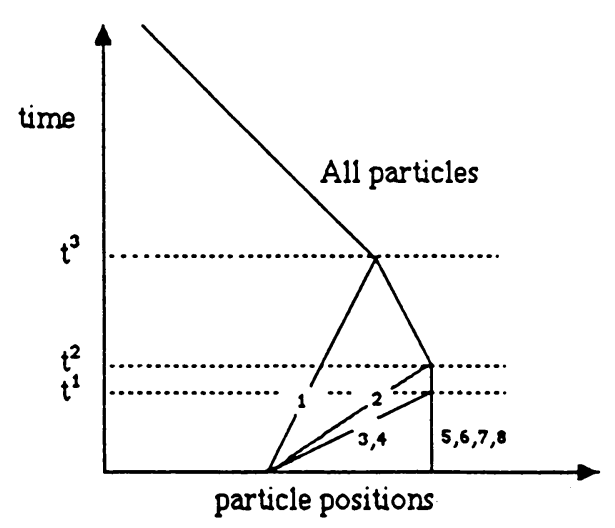

(b) Particle trajectories.

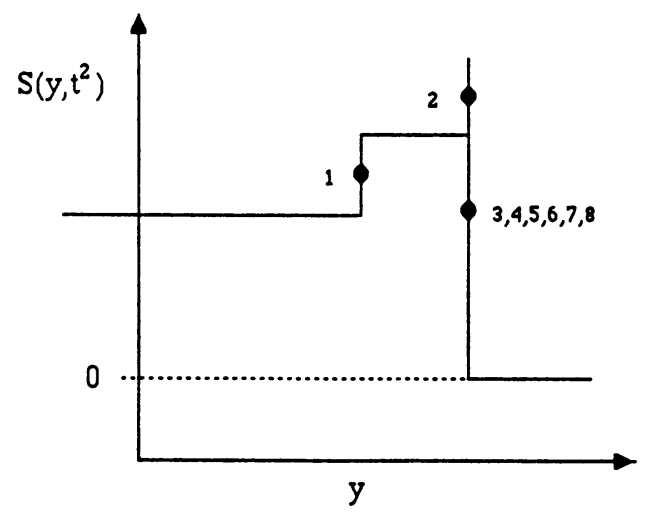

(d) Approximation at time $t^{2}$.

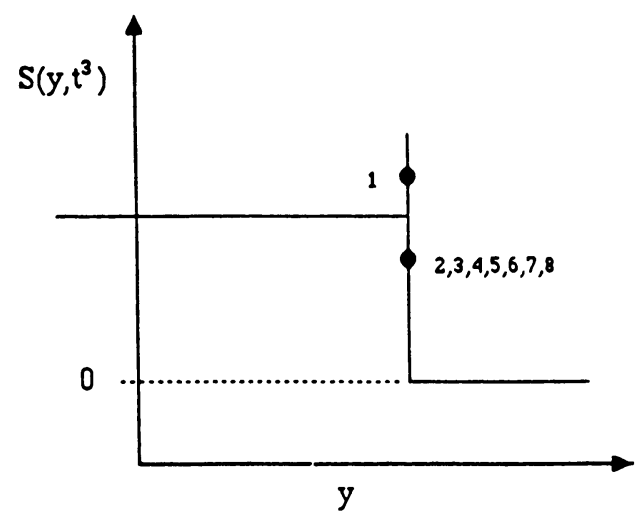

(e) Approximation at time $t^{3}$.

\section{FIGURE 1}

Approximate advection operator operating on 8 particles, particles 1, 2, 4 having positive strength, particles $3,5,6,7,8$ having negative strength. 
Let us observe the following facts:

(a) Since the particles have a finite maximum propagation speed, we conclude that $t^{k+1}>t^{k}$ for $k=0,1, \ldots$.

(b) For $0<t \leq t^{k}, k=1,2, \ldots$, every positive discontinuity is generated by one particle with positive strength. Equivalently, a positive discontinuity of strength greater than $h$ can only exist at time $t=0$.

(c) The distance between two adjacent discontinuities of positive strength increases as $t$ increases.

(d) The distance between a positive discontinuity and a nonpositive discontinuity only decreases with time if the strength of the nonpositive discontinuity is less than or equal to $-2 h$.

From statements (c) and (d) we conclude that the accumulated strength of the particles intersecting at a particular point at time $t^{k}, k=1,2, \ldots$, must be nonpositive. Hence at time $t^{k}$, at each point of intersection, nonpositive and positive discontinuities join to form a nonpositive discontinuity. So at time $t^{k}$, the number of positive and nonpositive discontinuities decreases. Since there are only a finite number of particles and so only a finite number of positive and nonpositive discontinuities initially, we conclude that there exists a $k$ such that $t^{k}=\infty$. Hence our approximation operator is defined for all $t>0$. Specifically,

$$
\tilde{A}_{t} S(y)=u^{L}+\sum_{i=1}^{m} H\left(y-x_{i}(t)\right) w_{i}
$$

where $S(y)=u^{L}+\sum_{i=1}^{m} H\left(y-x_{i}\right) w_{i}$.

We remark that the operator $\tilde{A}_{t}$ satisfies the semigroup property relative to the variable $t$. That is, for $t_{1}, t_{2} \geq 0$,

$$
\tilde{A}_{t_{1}} \tilde{A}_{t_{2}} S=\tilde{A}_{t_{1}+t_{2}} S \text {. }
$$

The particle positions at the $j+1 / 2$ fractional step of the method are given by $X_{i}^{j+1 / 2}=x_{i}(k)$, where $x_{i}(0)=X_{i}^{j}$, and the step function approximation is

$$
S^{j+1 / 2} u_{0}(y)=u^{L}+\sum_{i=1}^{m} H\left(y-X_{i}^{j+1 / 2}\right) w_{i}=\tilde{A}_{k} S^{j} u_{0}(y) .
$$

Step 3: Approximate Diffusion. The next step involves solving the diffusion equation. This is accomplished by adding a random component to the position of the particles. Let $\eta_{i}^{j+1}, i=1, \ldots, m$, be an independent collection of normally distributed random variables such that $E\left[\eta_{i}^{j+1}\right]=0$ and $\operatorname{Var}\left[\eta_{i}^{j+1}\right]=2 \nu k$. Then the new positions of the particles are given by

$$
X_{i}^{j+1}=X_{i}^{j+1 / 2}+\eta_{i}^{j+1}
$$

and the numerical approximation is given by

$$
S^{j+1} u_{0}(y)=u^{L}+\sum_{i=1}^{m} H\left(y-X_{i}^{j+1}\right) w_{i}=\tilde{D}_{k} S^{j+1 / 2} u_{0}(y) .
$$

Step 4. The final part of the fractional step involves setting $j:=j+1$ and going back to Step 2 if $j<n$. If $j=n$ then our numerical approximation for $F_{n k} u_{0}$ is 
given by

$$
S^{n} u_{0}(y)=u^{L}+\sum_{i=1}^{m} H\left(y-X_{i}^{n}\right) w_{i}=\left[\tilde{D}_{k} \tilde{A}_{k}\right]^{n} S^{0} u_{0}(y) .
$$

3. The Convergence Theorem. We are now in a position to discuss our convergence result. First, though, we make explicit some assumptions regarding the initial data $u_{0}$, the time step $k$, and the spatial parameter $h$, that will be used throughout the rest of the paper.

Assumption 1. We will assume that the initial data $u_{0} \in C^{2}(\mathbf{R})$ is constant in a neighborhood of infinity in the sense that there exist constants $K>0, u^{L}$, and $u^{R}$ such that

$$
\begin{aligned}
u_{0}(x)=u^{L} & \text { for } x<-K \\
=u^{R} & \text { for } x>K
\end{aligned}
$$

Assumption 2. We suppose that the time step satisfies $k<1 /(2 B)$, where

$$
B=\sup _{t \geq 0}\left\{\left\|F_{t} u_{0}\right\|_{L^{\infty}},\left\|\partial_{x} F_{t} u_{0}\right\|_{L^{\infty}},\left\|\partial_{x}^{2} F_{t} u_{0}\right\|_{L^{\infty}},\left\|\partial_{x} F_{t} u_{0}\right\|_{L^{1}}\right\}
$$

Assumption 3. We suppose that the spatial parameter satisfies $h \leq 1 / 3$ and that $h$ divides $u^{L}-u^{R}$.

Remark. The constant $B$ defined in Assumption 2 is finite. There exists an explicit formula for the solution of Burgers equation (Hopf [23], Cole [12]) from which bounds on the derivatives of the solution in terms of $\nu$ and the derivatives of $u_{0}$ can be obtained. The bound on the total variation of the solution, $\left\|\partial_{x} F_{t} u_{0}\right\|_{L^{1}} \leq$ $\left\|\partial_{x} u_{0}\right\|_{L^{1}}$, follows from Kružkov [25, p. 239].

It should be noted that $B$ has a $\nu^{-2}$ dependence. This follows from the long-time convergence of solutions of the viscous Burgers equation to travelling wave solutions of the form $f((x-c t) / \nu)$. The resultant restraint on the time step $\left(k<O\left(\nu^{2}\right)\right)$ is actually too restrictive and can be relaxed to $k<O(\nu)$ (see Roberts [31, Theorem 2.2.1]). We have chosen the more restrictive assumption so as to simplify the presentation.

Our convergence result can now be stated explicitly.

Convergence TheOrem. Let $u_{0}, k$ and $h$ satisfy Assumptions 1,2 and 3, respectively. Let $T$ be the final time, and suppose the time step and spatial parameters satisfy the relation $k=h^{1 / 4}$. Then for any positive integer $n$ such that $n k \leq T$ and any $\alpha>1$,

$$
P\left(\left\|F_{n k} u_{0}-\left[\tilde{D}_{k} \tilde{A}_{k}\right]^{n} S^{0} u_{0}\right\|_{L^{1}}>M_{1} \alpha h^{1 / 4}(\ln (1 / h))^{2}\right) \leq M_{2} h^{\alpha \ln (1 / h)-5 / 4}
$$

and

$$
E\left[\left\|F_{n k} u_{0}-\left[\tilde{D}_{k} \tilde{A}_{k}\right]^{n} S^{0} u_{0}\right\|_{L^{1}}\right] \leq C h^{1 / 4}(\ln (1 / h))^{2}
$$

where the constants $M_{1}, M_{2}$ and $\left(C^{\prime}\right.$ depend only on $u_{0}, \nu$ and $T$.

Remark. Here we use the notation $P(\cdot)$ to denote the probability of a particular event, and $E[\cdot]$ to denote the expected value of a particular random variable. 
The use of the $L^{1}$-norm as a measure of error arises in a completely natural way since all of the exact operators satisfy stability results in that norm. Namely,

$$
\begin{aligned}
\left\|F_{t} u-F_{t} v\right\|_{L^{1}} & \leq\|u-v\|_{L^{1}}, \\
\left\|A_{t} u-A_{t} v\right\|_{L^{1}} & \leq\|u-v\|_{L^{1}}, \\
\left\|D_{t} u-D_{t} v\right\|_{L^{1}} & \leq\|u-v\|_{L^{1}}
\end{aligned}
$$

for $u, v \in L_{\text {loc }}^{1}(\mathbf{R})$ such that $u-v \in L_{1}$ (see Kružkov [25]).

The proof of the convergence theorem is accomplished in five stages. The first three consist of analyzing the accuracy of the approximation operators $S_{0}, \tilde{A}_{k}$ and $\tilde{D}_{k}$, as contained in the following three theorems.

THEOREM 1. Let $u_{0}$ satisfy Assumption 1 and $h$ divide $u^{L}-u^{R}$. Then there exists a step function $S^{0} u_{0}$, generated by a collection of $m$ particles with positions $\left\{X_{i}^{0}\right\}_{i=1}^{m}$ and strengths $\left\{w_{i}\right\}_{i=1}^{m}$ which satisfy $\left|X_{i}^{0}\right| \leq K$ and $\left|w_{i}\right|=h$, so that $m h \leq\left\|\partial_{x} u_{0}\right\|_{L^{1}}$ and

$$
\left\|S^{0} u_{0}-u_{0}\right\|_{L^{1}} \leq C h
$$

where $C=2 K$ ( $K$ as in Assumption 1$)$.

A proof of this result can be found in Roberts [31, Chapter 1].

The accuracy of the operator $\tilde{A}_{k}$ is given by the following theorem, the proof of which is contained in Section 4.

THEOREM 2. Suppose we have $m$ particles with initial positions $\left\{x_{i}\right\}_{i=1}^{m}$ and strengths $\left\{w_{i}\right\}_{i=1}^{m}$ such that $\left|w_{i}\right|=h$. Let the following step function be given:

$$
S(y)=u^{L}+\sum_{i=1}^{m} H\left(y-x_{i}\right) w_{i}
$$

where $u^{L} \in \mathbf{R}$. Then for $t \geq 0$,

$$
\left\|A_{t} S-\tilde{A}_{t} S\right\|_{L^{1}} \leq \frac{1}{4} h^{2} m t
$$

In Section 5 the $\tilde{D}_{k}$ operator is shown to satisfy the following result.

THEOREM 3. Let $T$ be the final time. Then for nonnegative integers $j$ such that $(j+1) k \leq T$, and for $\alpha>1$,

$$
\begin{aligned}
& P\left(\left\|D_{k} S^{j+1 / 2} u_{0}-\tilde{D}_{k} S^{j+1 / 2} u_{0}\right\|_{L^{1}}>M_{1} \alpha h^{1 / 2}(\ln (1 / h))^{2}\right) \\
& \quad \leq M_{2} h^{\alpha \ln (1 / h)-1}
\end{aligned}
$$

and

$$
E\left[\left\|D_{k} S^{j+1 / 2} u_{0}-\tilde{D}_{k} S^{j+1 / 2} u_{0}\right\|_{L^{1}}\right] \leq C h^{1 / 2}(\ln (1 / h))^{2},
$$

where $S^{j+1 / 2} u_{0}$ is the random step function generated by the random variables $X_{i}^{j+1 / 2}$ and the constants $M_{1}, M_{2}$ and $C$ depend only on $u_{0}, \nu$ and $T$.

Remark. The proof of this result is based on the observation that

$$
\tilde{D}_{k} S^{j+1 / 2} u_{0}(y)
$$

can be represented as a sum of bounded random variables. Application of a result due to Hoeffding [22, Theorem 1] allows us to show that

$$
P\left(\left|D_{k} S^{j+1 / 2} u_{0}(y)-\tilde{D}_{k} S^{j+1 / 2} u_{0}(y)\right|>m h \delta\right) \leq 2 \exp \left(-2 \delta^{2} m\right) .
$$


This estimate can be extended to obtain an $L^{\infty}$ estimate in any bounded interval, which in turn leads to an $L^{1}$ estimate over any bounded interval. The result of Theorem 3 then follows by noting that there exists a bounded interval such that with high probability, the $L^{1}$ error outside that interval is small.

The fourth stage of the proof of the convergence theorem involves studying the accuracy of the viscous splitting algorithm, that is, the fractional step algorithm in which $\left[D_{k}, A_{k}\right]^{n}$ is used to approximate $F_{n k}$ (note that the exact operators $D_{k}$ and $A_{k}$ are used).

It would be unlikely that a fractional step algorithm using random walks would converge if the corresponding viscous splitting algorithm did not converge. In Section 6 of this paper we will prove that the method is justified when we show that the following theorem is true.

Viscous SPlitting TheOREM. Let $u_{0}$ and $k$ satisfy Assumptions 1 and 2, respectively. Then for $n \in \mathbf{Z}^{+}$,

$$
\left\|F_{n k} u_{0}-\left[D_{k} A_{k}\right]^{n} u_{0}\right\|_{L^{1}} \leq C n k^{2}
$$

where $C$ is a constant depending only on $u_{0}$ and $\nu$.

This result is not only a justification, it is also an integral part in the proof of the convergence theorem. By considering exact operators, we are able to use standard tools of analysis to obtain an estimate on the interaction of the advective and diffusive parts of the fractional step algorithm.

The proof of the viscous splitting theorem is based on the one-step estimate

$$
\left\|F_{k} v-\left[D_{k} A_{k}\right] v\right\|_{L^{1}} \leq C k^{2},
$$

when $v=F_{j k} v_{0}$ for $j=0, \ldots, n-1$. The viscous splitting theorem then follows from a simple summation of the one-step estimates.

Some mention should be made about the dependence of $C$ on $\nu$. Referring to Lemma 8 in Section 6 , we conclude that $C \leq 8 \nu B^{2}+31 \nu^{1 / 2} B^{5 / 2}$. Since $B$ is $O\left(\nu^{-2}\right)$, we have that $C$ is $O\left(\nu^{-9 / 2}\right)$, though a more detailed analysis (Roberts [31, $\S 2.3])$ shows that the optimal order is $\nu^{-1}$. On the other hand, one would expect that for fixed $n$ and $k$ the quantity $\left\|F_{n k} u_{0}-\left[D_{k} A_{k}\right]^{n} u_{0}\right\|_{L^{1}}$ would decrease with decreasing $\nu$. This is the observed numerical behavior. The resolution between these two observations is obtained by realizing that the full behavior of the error of the viscous splitting algorithm is associated with the interaction of the fractional steps over a number of steps and cannot be derived from a simple summation of the errors at each time step. This is in contrast to the results obtained by Beale and Majda [1] for the case of viscous splitting of the Navier-Stokes equation, where simple summation of the one time step errors produces the observed result, namely that the viscous splitting error is $O(\nu)$.

Proof of the Convergence Theorem. Having completed the analysis of the accuracy of the operators $S^{0}, \tilde{A}_{k}$ and $\tilde{D}_{k}$ and the viscous splitting algorithm, it is then necessary to combine the results to produce a proof of the convergence theorem. A simple triangle inequality argument shows that

$$
\left\|F_{n k} u_{0}-\left[\tilde{D}_{k} \tilde{A}_{k}\right] S^{0} u_{0}\right\|_{L^{1}}
$$


is less than or equal to

$$
\begin{gathered}
\left\|F_{n k} u_{0}-\left[D_{k} A_{k}\right]^{n} u_{0}\right\|_{L^{1}} \\
+\left\|\left[D_{k} A_{k}\right]^{n} u_{0}-\left[D_{k} A_{k}\right]^{n} S^{0} u_{0}\right\|_{L^{1}} \\
+\left\|\left[D_{k} A_{k}\right]^{n} S^{0} u_{0}-\left[\tilde{D}_{k} \tilde{A}_{k}\right]^{n} S^{0} u_{0}\right\|_{L^{1}}
\end{gathered}
$$

Expression (8) is just the error of the viscous splitting algorithm and so is estimated in the viscous splitting theorem.

A simple induction argument using the stability of the advection and diffusion operators (inequalities (6) and (7)), together with the accuracy estimate of the $S^{0}$ operator contained in Theorem 1 , shows that

$$
\left\|\left[D_{k} A_{k}\right]^{n} u_{0}-\left[D_{k} A_{k}\right]^{n} S^{0} u_{0}\right\|_{L^{1}} \leq\left\|u_{0}-S^{0} u_{0}\right\|_{L^{1}} \leq C h
$$

for some constant $C$.

Now expression (10) is bounded by the sum of terms

$$
\sum_{j=0}^{n-1}\left\|\left[D_{k} A_{k}\right]^{n-j-1} D_{k} A_{k} S^{j} u_{0}-\left[D_{k} A_{k}\right]^{n-j-1} D_{k} \tilde{A}_{k} S^{j} u_{0}\right\|_{L^{1}}
$$

and

$$
\sum_{j=0}^{n-1}\left\|\left[D_{k} A_{k}\right]^{n-j-1} D_{k} S^{j+1 / 2} u_{0}-\left[D_{k} A_{k}\right]^{n-j-1} \tilde{D}_{k} S^{j+1 / 2} u_{0}\right\|_{L^{1}}
$$

The stability of the advection and diffusion operators can again be used to show that the terms in expression (11) are less than or equal to

$$
\left\|A_{k} S^{j} u_{0}-\tilde{A}_{k} S^{j} u_{0}\right\|_{L^{1}}
$$

This estimate together with the accuracy of the advection operator $\tilde{A}_{k}$, as estimated in Theorem 2, implies that the terms in (11) are bounded by $\frac{1}{4} h^{2} m k$. Since $m h \leq$ $\left\|\partial_{x} u_{0}\right\|_{L^{1}}$ (Theorem 1) and $n k \leq T$, we conclude that the summation (11) is less than or equal to $\frac{1}{4}\left\|\partial_{x} u_{0}\right\|_{L^{1}} h T$.

The stability of the operators $A_{k}$ and $D_{k}$ also implies that expression (12) is bounded by the sum of random variables

$$
\sum_{j=0}^{n-1}\left\|D_{k} S^{j+1 / 2} u_{0}-\tilde{D}_{k} S^{j+1 / 2} u_{0}\right\|_{L^{1}}
$$

Combining these results, we conclude that if $u_{0}, k$ and $h$ satisfy Assumptions 1, 2 and 3, respectively, and if $n$ is a positive integer such that $n k \leq T$, then there exists a constant $C_{1}$ depending only on $u_{0}, \nu$ and $T$ such that

$$
\begin{aligned}
& \left\|F_{n k} u_{0}-\left[\tilde{D}_{k} \tilde{A}_{k}\right]^{n} S^{0} u_{0}\right\|_{L^{1}} \\
& \quad \leq C_{1}(h+k)+\sum_{j=0}^{n-1}\left\|D_{k} S^{j+1 / 2} u_{0}-\tilde{D}_{k} S^{j+1 / 2} u_{0}\right\|_{L^{1}} .
\end{aligned}
$$


The conclusion of the proof of the convergence theorem follows by an application of Theorem 3 to the terms in the sum in expression (13). In particular,

$$
\begin{aligned}
& P\left(\left\|F_{n k} u_{0}-\left[\tilde{D}_{k} \tilde{A}_{k}\right]^{n} S^{0} u_{0}\right\|_{L^{1}}>C_{1}(h+k)+n M_{1}^{1} \alpha h^{1 / 2}(\ln (1 / h))^{2}\right) \\
& \quad \leq P\left(\sum_{j=0}^{n-1}\left\|D_{k} S^{j+1 / 2} u_{0}-\tilde{D}_{k} S^{j+1 / 2} u_{0}\right\|_{L^{1}}>n M_{1}^{1} \alpha h^{1 / 2}(\ln (1 / h))^{2}\right) \\
& \quad \leq \sum_{j=0}^{n-1} P\left(\left\|D_{k} S^{j+1 / 2} u_{0}-\tilde{D}_{k} S^{j+1 / 2} u_{0}\right\|_{L^{1}}<M_{1}^{1} \alpha h^{1 / 2}(\ln (1 / h))^{2}\right) \\
& \quad \leq n M_{2}^{1} h^{\alpha \ln (1 / h)-1},
\end{aligned}
$$

where $M_{1}^{1}$ and $M_{2}^{1}$ are the constants contained in Theorem 3. Since we assume that $k=h^{1 / 4}$ and $h<\frac{1}{3}$, we conclude

$$
C_{1}(h+k)+n M_{1}^{1} \alpha h^{1 / 2}(\ln (1 / h))^{2} \leq M_{1} \alpha h^{1 / 4}(\ln (1 / h))^{2}
$$

and

$$
M_{2}^{1} n h^{\alpha \ln (1 / h)-1} \leq M_{2} h^{\alpha \ln (1 / h)-5 / 4},
$$

where the constants $M_{1}$ and $M_{2}$ depend only on $u_{0}, \nu$ and $T$.

Consequently, we conclude that

$$
P\left(\left\|F_{n k} u_{0}-\left[\tilde{D}_{k} \tilde{A}_{k}\right]^{n} S^{0} u_{0}\right\|_{L^{1}}>M_{1} \alpha h^{1 / 4}(\ln (1 / h))^{2}\right) \leq M_{2} h^{\alpha \ln (1 / h)-5 / 4}
$$

as required in the Convergence Theorem.

In a similar manner, we can estimate the expected value of the $L^{1}$ error of our numerical method. Expression (13) and Theorem 3 imply that

$$
\begin{aligned}
& E\left[\left\|F_{n k} u_{0}-\left[\tilde{D}_{k} \tilde{A}_{k}\right]^{n} S^{0} u_{0}\right\|_{L^{1}}\right] \\
& \quad \leq C_{1}(h+k)+\sum_{j=0}^{n-1} E\left[\left\|D_{k} S^{j+1 / 2} u_{0}-\tilde{D}_{k} S^{j+1 / 2} u_{0}\right\|_{L^{1}}\right] \\
& \quad \leq C_{1}(h+k)+C_{2} n h^{1 / 2}(\ln (1 / h))^{2},
\end{aligned}
$$

where $C_{2}$ is the constant contained in Theorem 3. Since $k=h^{1 / 4}, n k \leq T$ and $\ln (1 / h)>1\left(\right.$ since $\left.h<\frac{1}{3}\right)$, we conclude that

$$
C_{1}(h+k)+C_{2} n h^{1 / 2}(\ln (1 / h))^{2} \leq C h^{1 / 2}(\ln (1 / h))^{2},
$$

where $C$ depends only on $u_{0}, \nu$ and $T$. Hence,

$$
E\left[\left\|F_{n k} u_{0}-\left[\tilde{D}_{k} \tilde{A}_{k}\right]^{n} S^{0} u_{0}\right\|_{L^{1}}\right] \leq C h^{1 / 4}(\ln (1 / h))^{2} .
$$

This completes the proof of the convergence theorem.

4. Convergence of the Approximate Advection Operator. In this section we will first study the accuracy of the $\tilde{A}_{t}$ operator during the time in which the particles are noninteracting.

LEMMA 1. For any step function $S$ generated by $m$ particles with initial positions $\left\{x_{i}\right\}_{i=1}^{m}$ and strengths $\left\{w_{i}\right\}_{i=1}^{m}$ such that $\left|w_{i}\right|=h$, we have

$$
\left\|A_{t} S-\tilde{A}_{t} S\right\|_{L^{1}} \leq \frac{1}{4} h^{2} m t
$$


for $0 \leq t \leq t^{1}$ where $t^{1}$ is the first time of intersection for the particle trajectories which generate $\tilde{A}_{t} S$.

Proof. We will show that

$$
t^{*}=\sup \left\{t \leq t^{1}:\left\|\tilde{A}_{\tau} S-A_{\tau} S\right\|_{L^{1}} \leq \frac{1}{4} h^{2} m \tau \text { for all } 0 \leq \tau \leq t\right\}
$$

is equal to $t^{1}$.

Suppose, on the contrary, that $t^{*}<t^{1}$. Now the function $\tilde{A}_{t^{*}} S$ is a step function with a finite number of discontinuities. Consequently, there will exist a $\delta>0$ such that for all $t^{*}<t<t^{*}+\delta$ the function $A_{t-t^{*}} \tilde{A}_{t^{*}} S$ will be given by a collection of noninteracting Riemann problem solutions. Now, by construction, the difference between the approximate solution $\tilde{A}_{t-t^{*}} \tilde{A}_{t^{*}} S$ and $A_{t-t^{*}} \tilde{A}_{t^{*}} S$ will be due to the difference in approximating rarefaction waves of height $h$ with positive discontinuities of the same height, situated at the average position of the rarefaction wave (see Figure 2). These small rarefaction waves have width $\left(t-t^{*}\right) h$ at time $t$. We conclude that the $L^{1}$-norm error due to each particle generating a discontinuity with positive strength is $\frac{1}{4} h^{2}\left(t-t^{*}\right)$. Notice that our approximation of a shock wave is exact, at least for times $t$ such that $t^{*}<t<t^{*}+\delta$. If $N$ is the number of particles generating a discontinuity with positive strength, then

$$
\left\|\tilde{A}_{t-t^{*}} \tilde{A}_{t^{*}} S-A_{t-t^{*}} \tilde{A}_{t^{*}} S\right\|_{L^{1}} \leq \frac{1}{4} h^{2} N\left(t-t^{*}\right) \leq \frac{1}{4} h^{2} m\left(t-t^{*}\right) .
$$

This last result, together with the stability of the advection operator (Eq. (6)) and the triangle inequality, implies that

$$
\begin{aligned}
\| \tilde{A}_{t} S & -A_{t} S \|_{L^{1}} \\
& \leq\left\|\tilde{A}_{t-t^{*}} \tilde{A}_{t^{*}} S-A_{t-t^{*}} \tilde{A}_{t^{*}} S\right\|_{L^{1}}+\left\|A_{t-t^{*}} \tilde{A}_{t^{*}} S-A_{t-t^{*}} A_{t^{*}} S\right\|_{L^{1}} \\
& \leq \frac{1}{4} h^{2} m\left(t-t^{*}\right)+\left\|\tilde{A}_{t^{*}} S-A_{t^{*}} S\right\|_{L^{1}} .
\end{aligned}
$$

By assumption, $\left\|\tilde{A}_{t^{*}} S-A_{t^{*}} S\right\|_{L^{1}} \leq \frac{1}{4} h^{2} m t^{*}$, and so we conclude that there exists a $\delta>0$ such that

$$
\left\|\tilde{A}_{t} S-A_{t} S\right\|_{L^{1}} \leq \frac{1}{4} h^{2} m t
$$

for all $t<t^{*}+\delta$. This implies that $t^{*}+\delta \leq t^{*}$. Hence we must have that $t^{*}=t^{1}$, as required.

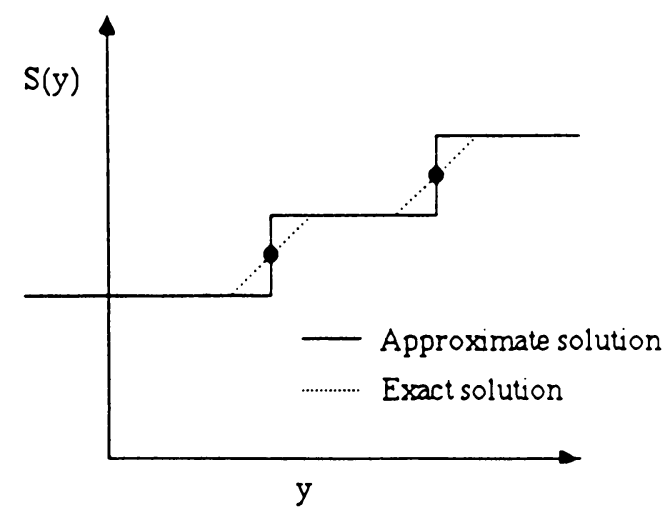

FIGURE 2

Comparison of the exact rarefaction wave solution and the approximate advection solution. 
Proof of Theorem 2. Lemma 1, together with a simple induction argument, can be used to prove Theorem 2 .

Suppose by induction that

$$
\left\|A_{t^{k}} S-\tilde{A}_{t^{k}} S\right\|_{L^{1}} \leq \frac{1}{4} h^{2} m t^{k}
$$

where $t^{k}$ is the $k$ th intersection time defined in Step 2 of Section 2 .

Let us suppose that $t^{k} \leq t \leq t^{k+1}$. Lemma 1 then implies that

$$
\left\|\tilde{A}_{t-t^{k}} \tilde{A}_{t^{k}} S-A_{t-t^{k}} \tilde{A}_{t^{k}} S\right\|_{L^{1}} \leq \frac{1}{4} h^{2} m\left(t-t^{k}\right) .
$$

Here we have applied Lemma 1 to the step function $\tilde{A}_{t^{k}} S$ and have made the observation that the time $t^{1}$ for initial data $\tilde{A}_{t^{k}} S$ is $t^{k+1}-t^{k}$ for the initial data $S$.

Consequently,

$$
\begin{aligned}
\| \tilde{A}_{t} S & -A_{t} S \|_{L^{1}} \\
& \leq\left\|\tilde{A}_{t-t^{k}} \tilde{A}_{t^{k}} S-A_{t-t^{k}} \tilde{A}_{t^{k}} S\right\|_{L^{1}}+\left\|A_{t-t^{k}} \tilde{A}_{t^{k}} S-A_{t-t^{k}} A_{t^{k}} S\right\|_{L^{1}} \\
& \leq \frac{1}{4} h^{2} m t
\end{aligned}
$$

where the first normed quantity is estimated by (16) and the second normed quantity is estimated by using the stability of $A_{t-t^{k}}$ together with assumption (15). The proof is completed by observing that the initial induction hypothesis for $k=0$ is obviously true.

5. The Random Walk Operator. We recall from Section 2 that the random particle positions $X_{i}^{j-1}$ and consequently the random step functions $S^{j-1} u_{0}$ depend on a collection of independent normally distributed random variables $\eta_{i}^{k}$, $i=1, \ldots, m, k=1, \ldots, j-1$. The random variables $X_{i}^{j-1 / 2}$ depend only on a deterministic mapping $\tilde{A}_{k}$ of the random positions $X_{i}^{j-1}$, and so depend on the random variables $\eta_{i}^{k}, \nu=1, \ldots, m, k=1, \ldots, j-1$. Let $\left(\Omega_{1}, \mathscr{F}_{1}, P_{1}\right)$ denote a probability space which supports the random variables $\eta_{i}^{k}, i=1, \ldots, m, k=1, \ldots, j-1$. Hence this probability space supports the random variables $X_{i}^{j-1}, X_{i}^{j-1 / 2}, \nu=1, \ldots, m$, and the random step functions $S^{j-1} u_{0}$ and $S^{j-1 / 2} u_{0}$.

The random variables $X_{i}^{j}$ are given by the relation

$$
X_{i}^{j}=X_{i}^{j-1 / 2}+\eta_{i}^{j}
$$

Let $\left(\Omega_{2}, \mathscr{F}_{2}, P_{2}\right)$ denote a probability space which supports the random variables $\eta_{i}^{j}$, $i=1, \ldots, m$. Thus the probability space $(\Omega, \mathscr{F}, P)$ on which the random variables $X_{i}^{j}$ are defined may be identified with the product space $\left(\Omega_{1} \times \Omega_{2}, \mathscr{F}_{1} \times \mathscr{F}_{2}, P_{1} \times P_{2}\right)$.

If we have a function $\Phi$ on $\Omega$, then we will use the notation $(\Phi)\left(\omega_{1}, \omega_{2}\right)$ to denote a specific realization of $\Phi$. In addition, $E_{1}$ and $E_{2}$ will denote integration over the spaces $\left(\Omega_{1}, \mathscr{F}_{1}, P_{1}\right)$ and $\left(\Omega_{2}, \mathscr{F}_{2}, P_{2}\right)$, respectively. This product structure allows us to calculate probability estimates and expected values involving the $X_{i}^{j}$ random variables by using Fubini's theorem on the space $\Omega=\Omega_{1} \times \Omega_{2}$.

We will use this structure to study the pointwise accuracy of our method.

LeMmA 2. For any $y \in \mathbf{R}$ and any $u_{0}$ satisfying Assumption 1 ,

$$
E_{2}\left[\tilde{D}_{k} S^{j-1 / 2} u_{0}(y)\right]=D_{k} S^{j-1 / 2} u_{0}(y) \text {. }
$$


Remark. Note that the quantity $D_{k} S^{j-1 / 2} u_{0}(y)$ is actually a function on $\Omega_{1}$. The proof of this lemma follows easily from the fact that

$$
E\left[H\left(z-\eta_{i}\right)\right]=D_{k} H(z)
$$

when $\eta_{i}$ is any normally distributed random variable with zero mean and variance $2 \nu k$.

To obtain an estimate of how close $\tilde{D}_{k} S^{j-1 / 2} u_{0}(y)$ is to $D_{k} S^{j-1 / 2} u_{0}(y)$, we make use of a corollary of the following theorem.

HoEfFding's THEOREM (Hoeffding [22, Theorem 1]). If $Z_{1}, \ldots, Z_{m}$ are $m$ independent random variables such that $0 \leq Z_{i} \leq 1, i=1, \ldots, m$, then for $\delta>0$,

$$
P\left(\frac{1}{m} \sum_{i=1}^{m} Z_{i}-\frac{1}{m} \sum_{i=1}^{m} E\left[Z_{i}\right]>\delta\right) \leq \exp \left(-2 \delta^{2} m\right) .
$$

COROLLARY. Let $Z_{i}, \ldots, Z_{m}$ be $m$ independent random variables such that for all $i=1, \ldots, m$ either $-1 \leq Z_{i} \leq 0$ or $0 \leq Z_{i} \leq 1$. Then, for $\delta>0$,

$$
P\left(\left|\frac{1}{m} \sum Z_{i}-\frac{1}{m} \sum E\left[Z_{i}\right]\right|>\delta\right) \leq 2 \exp \left(-2 \delta^{2} m\right) .
$$

This corollary is proved by applying Hoeffding's result to the sets of random variables $Y_{i}$ and $X_{i}$ where $Y_{i}=Z_{i}$ and $X_{i}=1-Z_{i}$ if $0 \leq Z_{i} \leq 1$, or $Y_{i}=1+Z_{i}$ and $X_{i}=-Z_{i}$ if $-1 \leq Z_{i} \leq 0$.

We can now study the accuracy of the random walk operator.

LEMMA 3. For any $y \in \mathbf{R}$ and $\delta>0$,

$$
P_{2}\left(\left|\tilde{D}_{k} S^{j-1 / 2} u_{0}(y)-D_{k} S^{j-1 / 2} u_{0}(y)\right|>m h \delta\right) \leq 2 \exp \left(-2 \delta^{2} m\right),
$$

where $j=1, \ldots, n$.

Remark. The quantity $P_{2}\left(\left|\tilde{D}_{k} S^{j-1 / 2} u_{0}(y)-D_{k} S^{j-1 / 2} u_{0}(y)\right|>m h \delta\right)$ is a function on $\Omega_{1}$. Since it is bounded by $2 \exp \left(-2 \delta^{2} m\right)$, we conclude that

$$
\begin{aligned}
& P\left(\left|\tilde{D}_{k} S^{j-1 / 2} u_{0}(y)-D_{k} S^{j-1 / 2} u_{0}(y)\right|>m h \delta\right) \\
& \quad=\int_{\Omega_{1}} P_{2}\left(\left|\tilde{D}_{k} S^{j-1 / 2} u_{0}(y)-D_{k} S^{j-1 / 2} u_{0}(y)\right|>m h \delta\right) P_{1}\left(d \omega_{1}\right)
\end{aligned}
$$

is also bounded by $2 \exp \left(-2 \delta^{2} m\right)$.

Proof of Lemma 3. Without loss of generality we may assume that the limit at minus infinity of $S^{j-1 / 2} u_{0}$ is zero $\left(u^{L}=0\right)$. For a fixed $\omega_{1} \in \Omega_{1}$, let us define $m$ independent random variables on $\Omega_{2}$ by $Z_{i}=H\left(y-X_{i}^{j-1 / 2}\left(\omega_{1}\right)-\eta_{i}^{j}\right) w_{i} / h$. Since $\left|w_{i}\right|=h$, we have that the $Z_{i}$ form an independent collection of random variables such that $0 \leq Z_{i} \leq 1$ or $-1 \leq Z_{i} \leq 0$. Also note that

$$
\frac{1}{m h} \tilde{D}_{k} S^{j-1 / 2} u_{0}(y)=\frac{1}{m} \sum_{i=1}^{m} Z_{i}
$$

and that

$$
\frac{1}{m h} D_{k} S^{j-1 / 2} u_{0}(y)=\frac{1}{m} \sum_{i=1}^{m} E_{1}\left[Z_{i}\right]
$$


as observed in Lemma 2 . Hence the preceding corollary implies that

$$
P_{2}\left(\left|\tilde{D}_{k} S^{j-1 / 2} u_{0}(y)-D_{k} S^{j-1 / 2} u_{0}(y)\right|>m h \delta\right) \leq 2 \exp \left(-2 \delta^{2} m\right) .
$$

This completes the analysis of the pointwise behavior of the random walk operator.

The $L^{1}$-norm behavior is estimated in two parts. First, in Lemma 4 we use the result of Lemma 3 to estimate the $L^{1}$ error over a finite region. The $L^{1}$ norm estimate over the full real line is completed by showing that the tail of the probability distribution of the particle positions $X_{i}^{j}$ decreases exponentially as the position tends to infinity. This in turn leads to an exponential estimate of the $L^{1}$-norm behavior of our step function solution outside a compact region.

LEMMA 4. Let $R>0$ and $\beta>1$. Then for $j=1, \ldots, n$,

$$
P\left(\left\|\tilde{D}_{k} S^{j-1 / 2} u_{0}-D_{k} S^{j-1 / 2} u_{0}\right\|_{L^{1}(-R, R)}>8 \beta R m^{1 / 2} h\right) \leq 2 m \exp \left(-2 \beta^{2}\right) .
$$

Proof. We will consider step functions $S_{1}$ and $S_{2}$ defined by

$$
S_{1}(y)=u^{L}+\sum_{w_{i}>0} H\left(y-X_{i}^{j-1 / 2}\right) w_{i}
$$

and

$$
S_{2}(y)=\sum_{w_{i}<0} H\left(y-X_{i}^{j-1 / 2}\right) w_{i}
$$

So $S_{1}$ is the step function generated by the particles with positive strengths and $S_{2}$ is the step function generated by the particles with negative strength. We let $m_{1}$ denote the number of particles with positive strength and $m_{2}$ be the number of particles with negative strength. We note that the operator $\tilde{D}_{k}$ has a natural definition for the data $S_{1}$ and $S_{2}$ so that $\tilde{D}_{k}\left(S_{1}+S_{2}\right)=\tilde{D}_{k} S^{j-1 / 2} u_{0}$ and so that the result of Lemma 3 holds with $S^{j-1 / 2} u_{0}$ and $m$ replaced by $S_{1}$ and $m_{1}$, or $S_{2}$ and $m_{2}$, respectively.

Let us first consider $S_{1}$ and suppose that $m_{1} \geq 1$. Since all of the particles generating $S_{1}$ have equal strength $h$, it follows that $\tilde{D}_{k} S_{1}(y)$ and $D_{k} S_{1}(y)$ are monotonically increasing functions of $y$ for fixed $\left(\omega_{1}, \omega_{2}\right) \in \Omega_{1} \times \Omega_{2}$.

For each $\omega_{1} \in \Omega_{1}$ we can choose a sequence of points $-R=a_{0}<a_{1}<\cdots<$ $a_{m_{1}}=R$ such that

$$
\left|D_{k} S_{1}\left(a_{i+1}\right)-D_{k} S_{1}\left(a_{i}\right)\right| \leq h .
$$

This follows from the fact that the range of $D_{k} S_{1}$ is contained in the interval $\left[u^{L}, u^{L}+m_{1} h\right]$ and the fact that $D_{k} S_{1}(y)$, for fixed $\omega_{1} \in \Omega_{1}$, is a smooth monotonic function of $y$. For each $i$ let

$$
\varepsilon_{i}=\left|\tilde{D}_{k} S_{1}\left(a_{i}\right)-D_{k} S_{1}\left(a_{i}\right)\right| .
$$

For the given fixed $\omega_{1} \in \Omega_{1}$ the quantity $\varepsilon_{i}$ is a random variable on $\Omega_{2}$ which gives a measure of the pointwise error at the point $a_{i}$. Now for $y \in\left[a_{i}, a_{i+1}\right]$, the monotonicity of $D_{k} S_{1}$ and $\tilde{D}_{k} S_{1}$ together with inequality (17) implies that

$$
\tilde{D}_{k} S_{1}(y)-D_{k} S_{1}(y) \leq \varepsilon_{i+1}+h
$$

and

$$
-\left(\tilde{D}_{k} S_{1}(y)-D_{k} S_{1}(y)\right) \leq \varepsilon_{i}+h
$$


and so

$$
\left|\tilde{D}_{k} S_{1}(y)-D_{k} S_{1}(y)\right| \leq \max \left(\varepsilon_{i}, \varepsilon_{i+1}\right)+h .
$$

This pointwise estimate allows us to conclude that

$$
\left\|\tilde{D}_{k} S_{1}-D_{k} S_{1}\right\|_{L^{1}(-R, R)} \leq 2 R\left[\max _{i}\left(\varepsilon_{i}\right)+h\right] .
$$

The function $\max _{i}\left(\varepsilon_{i}\right)$ for a given $\omega_{1} \in \Omega_{1}$ is a random variable on $\Omega_{2}$. Hence for $\delta>0$, Lemma 3 implies

$$
\begin{aligned}
P_{2}( & \left.\left\|\tilde{D}_{k} S_{1}-D_{k} S_{1}\right\|_{L^{1}(-R, R)}>2 R\left[m_{1} h \delta+h\right]\right) \\
& \leq P_{2}\left(2 R\left[\max _{i}\left(\varepsilon_{i}\right)+h\right]>2 R\left[m_{1} h \delta+h\right]\right)=P_{1}\left(\max _{i}\left(\varepsilon_{i}\right)>m_{1} h \delta\right) \\
& =P_{2}\left(\left|\tilde{D}_{k} S_{1}\left(a_{i}\right)-D_{k} S_{1}\left(a_{i}\right)\right|>m_{1} h \delta \text { for some } a_{i}\right) \\
& \leq \sum_{i=0}^{m_{1}} P_{2}\left(\left|\tilde{D}_{k} S_{1}\left(a_{i}\right)-D_{k} S_{1}\left(a_{i}\right)\right|>m_{1} h \delta\right) \\
& \leq 2 m_{1} \exp \left(-2 \delta^{2} m_{1}\right)
\end{aligned}
$$

(since $\left.m_{1}+1 \leq 2 m_{1}\right)$. Hence,

$$
P_{2}\left(\left\|\tilde{D}_{k} S_{1}-D_{k} S_{1}\right\|_{L^{1}(-R, R)}>2 R\left[m_{1} h \delta+h\right]\right) \leq 2 m_{1} \exp \left(-2 \delta^{2} m_{1}\right) .
$$

Now we have a conditional estimate on the random variable

$$
\left\|\tilde{D}_{k} S_{1}-D_{k} S_{1}\right\|_{L^{1}(-R, R)}
$$

from which we easily conclude that

$$
P\left(\left\|\tilde{D}_{k} S_{1}-\tilde{D}_{k} S_{1}\right\|_{L^{1}(-R, R)}>2 R h\left[m_{1} \delta+1\right]\right) \leq 2 m_{1} \exp \left(-2 \delta^{2} m_{1}\right)
$$

If we make the substitution $\delta=m_{1}^{-1 / 2} \beta$ and use the fact that $m_{1} \geq 1$ and $\beta>1$, then we deduce that

$$
P\left(\left\|\tilde{D}_{k} S_{1}-\tilde{D}_{k} S_{1}\right\|_{L^{1}(-R, R)}>4 \beta R m_{1}^{1 / 2} h\right) \leq 2 m_{1} \exp \left(-2 \beta^{2}\right) .
$$

A completely analogous result holds for $S_{2}$ with $m_{1}$ replaced by $m_{2}$. A simple triangle inequality argument then shows that

$$
P\left(\left\|\tilde{D}_{k} S^{j-1 / 2} u_{0}-D_{k} S^{j-1 / 2} u_{0}\right\|_{L^{1}(-R, R)}>8 \beta R m^{1 / 2} h\right) \leq 2 m \exp \left(-2 \beta^{2}\right)
$$

as required.

LEMMA 5. Assume that the step function $S^{0} u_{0}$ is generated by $m$ particles with positions which are contained in the interval $[-K, K]$. Suppose $n k \leq T$. For any $\gamma>0$ set $R=K+\left[\left\|u_{0}\right\|_{L^{\infty}}+m h\right] T+2 \gamma$. Then

$$
\begin{aligned}
& P\left(\left\|\tilde{D}_{k} S^{j-1 / 2} u_{0}-D_{k} S^{j-1 / 2} u_{0}\right\|_{L^{1}(-\infty,-R)}>\sqrt{\frac{\nu T}{\pi}} m h \exp \left(\frac{-\gamma^{2}}{4 \nu T}\right)\right) \\
& \leq \frac{2 m}{\gamma} \sqrt{\frac{\nu T}{\pi}} \exp \left(\frac{-\gamma^{2}}{4 \nu T}\right)
\end{aligned}
$$


and

$$
\begin{aligned}
& P\left(\left\|\tilde{D}_{k} S^{j-1 / 2} u_{0}-D_{k} S^{j-1 / 2} u_{0}\right\|_{L^{1}(R, \infty)}>\sqrt{\frac{\nu T}{\pi}} m h \exp \left(\frac{-\gamma^{2}}{4 \nu T}\right)\right) \\
& \leq \frac{2 m}{\gamma} \sqrt{\frac{\nu T}{\pi}} \exp \left(\frac{-\gamma^{2}}{4 \nu T}\right)
\end{aligned}
$$

for $j=1,2, \ldots, n$.

Proof. We will only prove statement (18), but note that statement (19) follows in a similar manner.

Let us suppose that the following claims are true.

Claim 1. If the random variables $X_{i}^{j-1 / 2}$ and $X_{i}^{j}$ are contained in $[-R+\gamma, \infty)$, then

$$
\left\|\tilde{D}_{k} S^{j-1 / 2} u_{0}-D_{k} S^{j-1 / 2} u_{0}\right\|_{L^{1}(-\infty,-R)} \leq \sqrt{\frac{\nu T}{\pi}} m h \exp \left(\frac{-\gamma^{2}}{4 \nu T}\right) .
$$

Claim 2. For all $i=1, \ldots, m$ and $j=1, \ldots, n$

$$
\begin{aligned}
& \text { (a) } P\left(X_{i}^{j-1 / 2} \leq-R+\gamma\right) \leq \frac{1}{\gamma} \sqrt{\frac{\nu T}{\pi}} \exp \left(\frac{-\gamma^{2}}{4 \nu T}\right) \\
& \text { (b) } P\left(X_{i}^{j} \leq-R+\gamma\right) \leq \frac{1}{\gamma} \sqrt{\frac{\nu T}{\pi}} \exp \left(\frac{-\gamma^{2}}{4 \nu T}\right)
\end{aligned}
$$

Claim 1 is equivalent to the statement that if

$$
\left\|\tilde{D}_{k} S^{j-1 / 2} u_{0}-D_{k} S^{j-1 / 2}\right\|_{L^{1}(-\infty,-R)}>\sqrt{\frac{\nu T}{\pi}} m h \exp \left(\frac{-\gamma^{2}}{4 \nu T}\right)
$$

then for some $i=1, \ldots, m, X_{i}^{j-1 / 2}<-R+\gamma$ or $X_{i}^{j}<-R+\gamma$. Consequently, if Claims 1 and 2 are true, then

$$
\begin{aligned}
& P\left(\left\|\tilde{D}_{k} S^{j-1 / 2} u_{0}-D_{k} S^{j-1 / 2}\right\|_{L^{1}(-\infty,-R)}>\sqrt{\frac{\nu T}{\pi}} m h \exp \left(\frac{-\gamma^{2}}{4 \nu T}\right)\right) \\
& \quad \leq P\left(X_{i}^{j-1 / 2}<-R+\gamma \text { or } X_{i}^{j}<-R+\gamma \text { for some } i=1, \ldots, m\right) \\
& \quad \leq \sum_{i=1}^{m}\left[P\left(X_{i}^{j-1 / 2}<-R+\gamma\right)+P\left(X_{i}^{j}<-R+\gamma\right)\right] \\
& \quad \leq \frac{2 m}{\gamma} \sqrt{\frac{\nu T}{\pi}} \exp \left(\frac{-\gamma^{2}}{4 \nu T}\right),
\end{aligned}
$$

which completes the proof of the lemma.

To complete the argument, though, we must prove that Claim 1 and Claim 2 are ture.

Proof of Claim 1. Let us consider the set

$$
E=\left\{\omega \in \Omega: X_{i}^{j}(\omega) \geq-R+\gamma \text { and } X_{i}^{j-1 / 2}(\omega) \geq-R+\gamma \text { for all } i=1, \ldots, m\right\} \text {. }
$$

For $\omega \in E$ and $y<-R, H\left(y-X_{i}^{j}(\omega)\right)=0$ and so

$$
\left(S^{j} u_{0}(y)\right)(\omega)=u^{L}+\sum_{i=1}^{m} H\left(y-X_{i}^{j}(\omega)\right)=u^{L}
$$


With the same restrictions on $\omega$ and $y$, we have that

$$
0 \leq H\left(y-X_{i}^{j-1 / 2}(\omega)\right) \leq H(y+R-\gamma) .
$$

A simple calculation then shows that for $\omega \in E$

$$
\begin{aligned}
\left\|D_{k} H\left(y-X_{i}^{j-1 / 2}\right)\right\|_{L^{1}(-\infty,-R)} & \leq\left\|D_{k} H(y+R-\gamma)\right\|_{L^{1}(-\infty,-R)} \\
& \leq \sqrt{\frac{\nu T}{\pi}} \exp \left(\frac{-\gamma^{2}}{4 \nu T}\right) .
\end{aligned}
$$

Consequently, for $\omega \in E$,

$$
\begin{gathered}
\left\|\tilde{D}_{k} S^{j-1 / 2} u_{0}-D_{k} S^{j-1 / 2} u_{0}\right\|_{L^{1}(-\infty,-R)}=\left\|u^{L}-D_{k} S^{j-1 / 2} u_{0}\right\|_{L^{1}(-\infty,-R)} \\
\leq h \sum_{i=1}^{m}\left\|D_{k} H\left(y-X_{i}^{j-1 / 2}\right)\right\|_{L^{1}(-\infty,-R)} \leq \sqrt{\frac{\nu T}{\pi}} m h \exp \left(\frac{-\gamma^{2}}{4 \nu T}\right)
\end{gathered}
$$

as required.

Proof of Claim 2. First observe that the random variables $X_{i}^{j-1 / 2}$ and $X_{i}^{j}$ can be written in the form

$$
X_{i}^{j-1 / 2}=\sum_{l=1}^{j-1} \eta_{i}^{l}+\sum_{l=0}^{j-1}\left(X_{i}^{l+1 / 2}-X_{i}^{l}\right)+X_{i}^{0}
$$

and

$$
X_{i}^{j}=\sum_{l=1}^{j} \eta_{i}^{l}+\sum_{l=0}^{j-1}\left(X_{i}^{l+1 / 2}-X_{i}^{l}\right)+X_{i}^{0}
$$

Referring back to Section 3 , we recall that $X_{i}^{l+1 / 2}=x_{i}(k)$ where $x_{i}(t)$ satisfies the evolution equation (4) with initial conditions $X_{i}^{l}$. It is easy to see that the approximate advection operator $\tilde{A}_{k}$ has a finite speed of propagation bounded by $\left\|u_{0}\right\|_{L^{\infty}}+m h$, so that

$$
\left|X_{i}^{l+1 / 2}-X_{i}^{l}\right| \leq k\left[\left\|u_{0}\right\|_{L^{\infty}}+m h\right] .
$$

Also, by assumption, $\left|X_{i}^{0}\right|<K$. Consequently, expressions (20) and (21) imply that

$$
X_{i}^{j-1 / 2} \geq \sum_{l=1}^{j-1} \eta_{i}^{l}-\left(j k\left[\left\|u_{0}\right\|_{L^{\infty}}+m h\right]+K\right) \geq \sum_{l=1}^{j-1} \eta_{i}^{l}-R+2 \gamma
$$

and

$$
X_{i}^{j} \geq \sum_{l=1}^{j} \eta_{i}^{l}-\left(j k\left[\left\|u_{0}\right\|_{L^{\infty}}+m h\right]+K\right) \geq \sum_{l=1}^{j} \eta_{i}^{l}-R+2 \gamma .
$$

The quantities $\sum_{l=1}^{j-1} \eta_{i}^{l}$ and $\sum_{l=1}^{j} \eta_{i}^{l}$ are sums of independent normally distributed random variables with zero mean and variance $2 \nu k$ and so are themselves normally distributed with mean zero and with variances $2 \nu k(j-1)$ and $2 \nu k j$, respectively. Since $(j-1) k \leq T$,

$$
\begin{aligned}
& P\left(X_{i}^{j-1 / 2} \leq-R+\gamma\right) \\
& \quad \leq P\left(\sum_{l=1}^{j-1} \eta_{i}^{l} \leq-\gamma\right) \leq \frac{1}{\gamma} \sqrt{\frac{\nu T}{\pi}} \exp \left(\frac{-\gamma^{2}}{4 \nu T}\right)
\end{aligned}
$$


and similarly

$$
P\left(X_{i}^{j} \leq-R+\gamma\right) \leq \frac{1}{\gamma} \sqrt{\frac{\nu T}{\pi}} \exp \left(\frac{-\gamma^{2}}{4 \nu T}\right) .
$$

Here we have used the fact that for a normally distributed random variable $\eta$ with variance $\sigma^{2}$ and zero mean,

$$
P(\eta \leq-\gamma) \leq \frac{1}{\gamma} \frac{\sigma}{\sqrt{2 \pi}} \exp \left(\frac{-\gamma^{2}}{2 \sigma^{2}}\right)
$$

(see Feller [14, p. 175]).

Proof of Theorem 3. We are now in a position to combine the results of Lemmas 4 and 5 to obtain a proof of Theorem 3, the theorem which specifies the accuracy of the random walk operator $\tilde{D}_{k}$.

First we will appiy Lemma 5 with the choice $\gamma=(4 \nu T)^{1 / 2} \alpha^{1 / 2} \ln (1 / h)$. Our assumption on the spatial parameter $h$ implies that $\ln (1 / h)>1$. In addition we assume $\alpha>1$. Consequently,

$$
\sqrt{\frac{\nu T}{\pi}} m h \exp \left(\frac{-\gamma^{2}}{4 \nu T}\right) \leq a_{1} \alpha h^{1 / 2}(\ln (1 / h))^{2}
$$

and

$$
\frac{2 m}{\gamma} \sqrt{\frac{\nu T}{\pi}} \exp \left(\frac{-\gamma^{2}}{4 \nu T}\right) \leq b_{1} h^{\alpha \ln (1 / h)-1},
$$

where $a_{1}=(\sqrt{\nu T / \pi}) m h$ and $b_{1}=m h / \sqrt{\pi}$. Lemma 5 then implies that

$$
\begin{aligned}
& P\left(\left\|\tilde{D}_{k} S^{j-1 / 2} u_{0}-D_{k} S^{j-1 / 2} u_{0}\right\|_{L^{1}(-\infty,-R)}>a_{1} \alpha h^{1 / 2}(\ln (1 / h))^{2}\right) \\
& \quad \leq b_{1} h^{\alpha \ln (1 / h)-1}
\end{aligned}
$$

and

$$
\begin{aligned}
& P\left(\left\|\tilde{D}_{k} S^{j-1 / 2} u_{0}-D_{k} S^{j-1 / 2} u_{0}\right\|_{L^{1}(R, \infty)}>a_{1} \alpha h^{1 / 2}(\ln (1 / h))^{2}\right) \\
& \quad \leq b_{1} h^{\alpha \ln (1 / h)-1},
\end{aligned}
$$

where $R=K+\left[\left\|u_{0}\right\|_{L^{\infty}}+m h\right] T+(16 \nu T)^{1 / 2} \alpha^{1 / 2} \ln (1 / h)$. With this choice of $R$ and with $\beta=\pi^{-1 / 2} \alpha^{1 / 2} \ln (1 / h)$, we observe that

$$
8 \beta R m^{1 / 2} h \leq a_{2} \alpha h^{1 / 2}(\ln (1 / h))^{2}
$$

and

$$
2 m \exp \left(-2 \beta^{2}\right) \leq b_{2} h^{\alpha \ln (1 / h)-1}
$$

where $a_{2}=(32 m h)^{1 / 2}\left(K+\left[\left\|u_{0}\right\|_{L^{\infty}}+m h\right] T+(16 \nu T)^{1 / 2}\right)$ and $b_{2}=m h$. Lemma 4 then implies that

$$
\begin{aligned}
& P\left(\left\|\tilde{D}_{k} S^{j-1 / 2} u_{o}-D_{k} S^{j-1 / 2} u_{0}\right\|_{L^{1}(-R, R)}>a_{2} \alpha h^{1 / 2}(\ln (1 / h))^{2}\right) \\
& \quad \leq b_{2} h^{\alpha \ln (1 / h)-1} .
\end{aligned}
$$

The triangle inequality and the estimates (22), (23) and (24) imply

$$
P\left(\left\|\tilde{D}_{k} S^{j-1 / 2} u_{0}-D_{k} S^{j-1 / 2} u_{0}\right\|_{L^{1}}>M_{1} \alpha^{1 / 2}(\ln (1 / h))^{2}\right) \leq M_{2} h^{\alpha \ln (1 / h)-1},
$$

where $M_{1}=2 a_{1}+a_{2}$ and $M_{2}=2 b_{1}+b_{2}$ are constants which depend only on $u_{0}$, $\nu$ and $T$. This completes the first part of Theorem 3. 
The estimation of the expected value

$$
E\left[\left\|\tilde{D}_{k} S^{j-1 / 2} u_{0}-D_{k} S^{j-1 / 2} u_{0}\right\|_{L^{1}}\right]
$$

is obtained by using the result that for a nonnegative random variable $Z, E[Z] \leq$ $a\left[1+\sum_{j=1}^{\infty} P(Z \geq j a)\right]$ (see Chung [11, Theorem 3.2.1]). Hence,

$$
\begin{aligned}
& E\left[\left\|\tilde{D}_{k} S^{j-1 / 2} u_{0}-D_{k} S^{j-1 / 2} u_{0}\right\|_{L^{1}}\right] \\
& \leq M_{1} h^{1 / 2}(\ln (1 / h))^{2}\left[1+\sum_{j=1}^{\infty} P\left(\left\|\tilde{D}_{k} S^{j-1 / 2} u_{0}-S_{k} S^{j-1 / 2} u_{0}\right\|_{L^{1}}\right.\right. \\
& \left.\left.\quad>j M_{1} h^{1 / 2}(\ln (1 / h))^{2}\right)\right] \\
& \leq M_{1} h^{1 / 2}(\ln (1 / h))^{2}\left[1+M_{2} \sum_{j=1}^{\infty} h^{j \ln (1 / h)-1] .}\right.
\end{aligned}
$$

By assumption, $h<\frac{1}{3}$, and so $h^{\ln (1 / h)}<\frac{3}{10}$ and $h^{\ln (1 / h)-1}<\frac{9}{10}$. Hence,

$$
\sum_{j=1}^{\infty} h^{j \ln (1 / h)-1}=\frac{h^{\ln (1 / h)-1}}{1-h^{\ln (1 / h)}} \leq 2 .
$$

This estimate implies that

$$
E\left[\left\|\tilde{D}_{k} S^{j-1 / 2} u_{0}-D_{k} S^{j-1 / 2} u_{0}\right\|_{L^{1}}\right] \leq C h^{1 / 2}(\ln (1 / h))^{2}
$$

where $C=M_{1}\left[1+2 M_{2}\right]$ is a constant which depends on $u_{0}, \gamma$ and $T$. This completes the proof of Theorem 3 .

6. The Viscous Splitting Algorithm. In this section we will prove the Viscous Splitting Theorem (see Section 3) by showing that the error arising from one time step of the viscous splitting algorithm is of order $k^{2}$, where $k$ is the time step. In particular, in Lemma 8 we show that

$$
\left\|F_{k} v-D_{k} A_{k} v\right\|_{L^{1}} \leq C k^{2}
$$

where the constant $C$ depends on $v, \nu$ but is independent of $k$. To obtain this bound, we use a result (Lemma 6 ) which uses a technique very similar to that used by Kružkov [25, p. 239] to show that the operator $F_{k}$ is stable in the $L^{1}$-norm, that is $\left\|F_{k} u-F_{k} v\right\|_{L^{1}} \leq\|u-v\|_{L^{1}}$.

LEMMA 6. Let $\nu$ and $T$ be fixed positive constants and let $a(x, t)$ and $b(x, t)$ be bounded continuous functions on $\Omega=\mathbf{R} \times[0, T]$ such that $b \in L^{1}(\Omega)$. Suppose that $w(x, t)$ is a solution of the equation

$$
\partial_{t} w+\partial_{x}(a w)+b=\nu \partial_{x}^{2} w, \quad w(x, 0)=w_{0}(x)
$$

where $w$ is an integrable bounded function in $C^{1}(\Omega)$ such that $\partial_{x} w$ is bounded. Then $w$ satisfies the inequality

$$
\int_{-\infty}^{\infty}|w(x, t)| d x \leq \int_{-\infty}^{\infty}\left|w_{0}(x)\right| d x+\int_{0}^{t} \int_{-\infty}^{\infty}|b(x, s)| d x d s
$$

for $0 \leq t \leq T$. 
Proof. Let us choose an arbitrary smooth bounded function $g(x, s)$ on $\Omega$ which has compact support. If we multiply Eq. (25) by $g$ and integrate over $\Omega$, then integration by parts implies that

$$
\begin{aligned}
\int_{-\infty}^{\infty} & w(x, t) g(x, t) d x-\int_{0}^{t} \int_{-\infty}^{\infty} L g(x, s) w(x, s) d x d s \\
= & \int_{-\infty}^{\infty} w_{0}(x) g(x, 0) d x-\int_{0}^{t} \int_{-\infty}^{\infty} g(x, s) b(x, s) d x d s
\end{aligned}
$$

where $L g=\partial_{t} g+a \partial_{x} g+\nu \partial_{x}^{2} g$. We want to extend this result to functions $g$ which satisfy $L g(x, s)=0,(x, s) \in \mathbf{R} \times[0, t]$, and have bounded compactly supported initial data at time $t$ (we will be solving backwards in time). Such a function will not be compactly supported on $\mathbf{R} \times[0, t]$, but will decrease exponentially to zero as $|x|$ tends to infinity (see Kružkov [25, Lemma 4]), so that there will exist constants $C_{1}, C_{2}>0$ such that $g(x, s) \leq C_{1} \exp \left(-C_{2}|x|\right)$ for $(x, s) \in \mathbf{R} \times[0, t]$.

We will show that Eq. (26) holds for functions $g$ which decrease exponentially to zero as $|x| \rightarrow \infty$. For a given parameter $R>0$ let $\eta=\psi * \chi_{[-R, R]}$, where $\psi$ is a smooth positive function with support in $[-1,1]$ such that $\int \psi=1, \chi_{[-R, R]}$ is the characteristic function of the region $[-R, R]$ and $*$ denotes convolution. If we apply expression (26) to the function $g \eta$, then

$$
\begin{aligned}
\left.\int_{-\infty}^{\infty} w g \eta\right|_{s=t} d x-\int_{0}^{t} \int_{-\infty}^{\infty} L g \eta w d x d s \\
\quad-\int_{0}^{t} \int_{-\infty}^{\infty}\left[w a \partial_{x} \eta-2 \nu \partial_{x} w \partial_{x} \eta+\nu w \partial_{x}^{2} \eta\right] g d x d s \\
=\left.\int_{-\infty}^{\infty} g w_{0} \eta\right|_{s=0} d x-\int_{0}^{t} \int_{-\infty}^{\infty} g \eta b d x d s
\end{aligned}
$$

We note that the term

$$
\left[w a \partial_{x} \eta-2 \nu \partial_{x} w \partial_{x} \eta+\nu w \partial_{x}^{2} \eta\right]
$$

is bounded and has support contained in two compact regions centered about the points $-R$ and $R$. Here we have used the assumptions that $\|w\|_{L^{\infty}}$ and $\left\|\partial_{x} w\right\|_{L^{\infty}}$ are bounded.

Since $g$ is assumed to decrease to zero exponentially as $|x|$ approaches infinity, it follows that

$$
\lim _{R \rightarrow \infty} \int_{0}^{t} \int_{-\infty}^{\infty}\left[w a \partial_{x} \eta-2 \nu \partial_{x} w \partial_{x} \eta+\nu w \partial_{x}^{2} \eta\right] g d x d s=0 .
$$

Obviously, $g \eta$ converges pointwise to the integrable function $g$ as $R \rightarrow \infty$. Hence the Lebesgue dominated convergence theorem can be applied to expression (27) to show that $g$ satisfies Eq. (26) provided $L g$ is integrable.

Choose an $r>0$ and let $\beta(x)=\operatorname{sgn}(w(x, t)) \chi_{[-r, r]}(x)$, where $\operatorname{sgn}(y)=1$ if $y>0$, 0 if $y=0$, and -1 if $y<0$. We will now mollify the function $\beta$. Specifically, for a smooth positive function $\psi$ with support contained in $[-1,1]$ we define $\psi^{\alpha}$ by the relation

$$
\psi^{\alpha}(x)=\frac{1}{\alpha} \psi\left(\frac{x}{\alpha}\right)
$$


and define $\beta^{\alpha}=\psi^{\alpha} * \beta$. Note that $\beta^{\alpha}$ is smooth and has compact support. Let $g^{\alpha}(x, t)$ be the unique bounded solution of the linear parabolic equation $L g^{\alpha}=0$ in $\mathbf{R} \times[0, t]$ with initial conditions $g^{\alpha}(x, t)=\beta^{\alpha}(x)$, where we solve backwards in time. As mentioned earlier, $g^{\alpha}$ will decrease exponentially to zero as $|x| \rightarrow \infty$ and so expression (26) will be satisfied with $g$ replaced by $g^{\alpha}$.

Now the maximum principle (Friedman [16, p. 34]) implies that for $0 \leq s \leq t$,

$$
\left|g^{\alpha}(x, s)\right| \leq \sup _{x \in \mathbf{R}}\left|g^{\alpha}(x, t)\right| \leq\left\|\beta^{\alpha}\right\|_{L^{\infty}} \leq 1
$$

Equations (26) and (28), together with the assumptions that $\left\|w_{0}\right\|_{L^{1}}$ and $\|b\|_{L^{1}}$ are finite, imply that

$$
\left|\int_{-\infty}^{\infty} w(x, t) g^{\alpha}(x, t) d x\right| \leq \int_{-\infty}^{\infty}\left|w_{0}(x)\right| d x+\int_{0}^{t} \int_{-\infty}^{\infty}|b(x, s)| d x d s .
$$

The function $\beta$ is a bounded measurable function and so it follows that $\beta^{\alpha}(x)$ converges to $\beta(x)$ as $\alpha$ tends to zero, for each Lebesgue point, $x$, of the function $\beta$ (Kružkov [25, p. 221]). The set of non-Lebesgue points of $\beta$ has zero measure and so $\beta^{\alpha}$ converges to $\beta$ pointwise almost everywhere on $\mathbf{R}$. That is, $g^{\alpha}(x, t)$ converges to $\beta(x)$ pointwise almost everywhere on $\mathbf{R}$. Hence the Lebesgue dominated convergence theorem implies that

$$
\int_{-r}^{r}|w(s, t)| d x \leq \int_{-\infty}^{\infty}\left|w_{0}(x)\right| d x+\int_{0}^{t} \int_{-\infty}^{\infty}|b(x, s)| d x d s .
$$

Finally, the proof of the lemma is concluded by applying the monotone convergence theorem as $r \rightarrow \infty$.

The next lemma is a technical result that is needed in Lemma 8.

LEMMA 7. Let $f, g \in C^{1}(\mathbf{R})$.

(a) If $\|f\|_{L^{\infty}}<\infty$ and $\left\|\partial_{x} g\right\|_{L^{1}}<\infty$ then

$$
\left\|D_{t} f D_{t} g-D_{t}(f g)\right\|_{L^{1}} \leq 2(\nu t)^{1 / 2}\|f\|_{L^{\infty}}\left\|\partial_{x} g\right\|_{L^{1}} .
$$

(b) If $\|f\|_{L^{1}}<\infty$ and $\left\|\partial_{x} g\right\|_{L^{\infty}}<\infty$ then

$$
\left\|D_{t} f D_{t} g-D_{t}(f g)\right\|_{L^{1}} \leq 2(\nu t)^{1 / 2}\|f\|_{L^{1}}\left\|\partial_{x} g\right\|_{L^{\infty}} .
$$

Proof. Let us introduce the notation

$$
G_{s}=\frac{1}{(2 \pi s)^{1 / 2}} \exp \left(-\frac{x^{2}}{2 s}\right)
$$

and observe that $D_{t}$ is equivalent to convolution by the function $G_{2 \nu t}$. In addition, note that

$$
\int_{-\infty}^{\infty} G_{s}\left(x-y_{2}\right) d y_{2}=1
$$

By explicitly writing out the operation of $D_{t}$ in terms of integrals, we see that

$$
\begin{aligned}
& \left\|D_{t} f D_{t} g-D_{t}(f g)\right\|_{L^{1}} \\
& =\int_{-\infty}^{\infty} \mid \int_{-\infty}^{\infty} \int_{-\infty}^{\infty} G_{2 \nu t}\left(x-y_{1}\right) G_{2 \nu t}\left(x-y_{2}\right) f\left(y_{1}\right) \\
& \quad \times\left[g\left(y_{2}\right)-g\left(y_{1}\right)\right] d y_{1} d y_{2} \mid d x
\end{aligned}
$$


Using the fact that $\left|f\left(y_{1}\right)\right| \leq\|f\|_{L^{\infty}}$, together with an application of the integral mean value theorem and an appropriate change of variables, part (a) of the lemma is reduced to an estimation of the quantity

$$
\|f\|_{L^{\infty}}\left\|\partial_{x} g\right\|_{L^{1}} \int_{-\infty}^{\infty} \int_{-\infty}^{\infty} G_{2 \nu t}\left(y_{1}\right) G_{2 \nu t}\left(y_{2}\right)\left|y_{2}-y_{1}\right| d y_{1} d y_{2}
$$

which by an explicit calculation is equal to $(8 \nu t / \pi)^{1 / 2}\|f\|_{L^{\infty}}\left\|\partial_{x} g\right\|_{L^{1}}$ and so is less than or equal to $2(\nu t)^{1 / 2}\|f\|_{L^{\infty}}\left\|\partial_{x} g\right\|_{L^{1}}$. Part (b) follows in a similar manner, but uses the differential form of the mean value theorem.

We are now ready to prove that the viscous splitting algorithm produces an error of order $k^{2}$ in one time step of size $k$.

LEMMA 8. Let $v_{0} \in C^{2}(\mathbf{R})$ be such that all derivatives up to second order and $\left\|\partial_{x} v_{0}\right\|_{L^{1}}$ are uniformly bounded by a constant $B$. Let $0 \leq k \leq 1 /(2 B)$. Then

$$
\left\|F_{k} v_{0}-D_{k} A_{k} v_{0}\right\|_{L^{1}} \leq C k^{2}
$$

where $C$ depends on $B$ and $\nu$.

Proof. We will show that Lemma 6 can be used to analyze the function

$$
w(x, t)=F_{t} v_{0}(x)-D_{t} A_{t} v_{0}(x) .
$$

Let us introduce some notation. Let $u=F_{t} v_{0}$ and $v=A_{t} v_{0}$. Using the notation introduced in the proof of the last lemma, we write the function $D_{t} A_{t} v_{0}$ as $G_{2 \nu t} * v$. Then

$$
w(x, t)=u(x, t)-G_{2 \nu t} * v(x, t) .
$$

An elementary calculation (John [24, p. 18]) shows that the solution of the inviscid Burgers equation, $v(x, t)$, for $0 \leq t \leq \frac{1}{2}\left\|\partial_{x} v_{0}\right\|_{L^{\infty}}^{-1}$ satisfies

$$
\|v\|_{L^{\infty}} \leq\left\|v_{0}\right\|_{L^{\infty}}, \quad\left\|\partial_{x} v\right\|_{L^{\infty}} \leq 2\left\|\partial_{x} v_{0}\right\|_{L^{\infty}}, \quad\left\|\partial_{x}^{2} v\right\|_{L^{\infty}} \leq 8\left\|\partial_{x}^{2} v_{0}\right\|_{L^{\infty}} .
$$

In addition, the stability of the inviscid Burgers equation (see Kružkov [25, p. 223]) implies $\left\|\partial_{x} v\right\|_{L^{1}} \leq\left\|\partial_{x} v_{0}\right\|_{L^{1}}$. Hence for $0 \leq k \leq 1 /(2 B)\left(\leq \frac{1}{2}\left\|\partial_{x} v_{0}\right\|_{L^{\infty}}^{-1}\right)$

$$
\|v\|_{L^{\infty}} \leq B, \quad\left\|\partial_{x} v\right\|_{L^{\infty}} \leq 2 B, \quad\left\|\partial_{x}^{2} v\right\|_{L^{\infty}} \leq 8 B, \quad\left\|\partial_{x} v\right\|_{L^{1}} \leq B .
$$

Now since $u$ satisfies the viscous Burgers equation and $G_{2 \nu t}(x)$, as a function of $x$ and $t$, satisfies the diffusion equation, it follows that for $0<t<1 /(2 B)$ the function $w$ satisfies the equation

$$
\partial_{t} w+\partial_{x}(a w)+b=\nu \partial_{x}^{2} w, \quad w(x, 0)=0,
$$

where $b=\left[\left(G_{2 \nu t}^{\gamma} * v\right)\left(G_{2 \nu t}^{\gamma} * \partial_{x} v\right)-G_{2 \nu t} *\left(v \partial_{x} v\right)\right]$ and $a=\frac{1}{2}\left[u+G_{2 \nu t} * v\right]$.

The conditions of Lemma 6 are satisfied provided $b \in L^{1}(\Omega)$. In that case we have

$$
\|w(\cdot, k)\|_{L^{1}} \leq \int_{0}^{k} \int_{-\infty}^{\infty}|b(x, t)| d x d t .
$$

An estimate for $\int_{-\infty}^{\infty}|b(x, t)| d x$ is obtained by applying Lemma 6 again. We first observe that in the region $\mathbf{R} \times[0, k]$ the function $b$ satisfies the equation

$$
\partial_{t} b+\partial_{x}(c b)+d=\nu \partial_{x}^{2} b, \quad b(x, 0)=0
$$


with $c=-G_{2 \nu t} * v$ and $d=d_{1}+d_{2}+d_{3}$, where

$$
\begin{aligned}
& d_{1}=2 \nu\left(G_{2 \nu t} * \partial_{x} v\right)\left(G_{2 \nu t} * \partial_{x}^{2} v\right), \\
& d_{2}=2\left[\left(G_{2 \nu t} * v\right)\left(G_{2 \nu t} * \partial_{x} v\right)^{2}-G_{2 \nu t} * v\left(\partial_{x} v\right)^{2}\right], \\
& d_{3}=\left[\left(G_{2 \nu t} * v\right)^{2}\left(G_{2 \nu t} * \partial_{x}^{2} v\right)-G_{2 \nu t} *\left(v^{2} \partial_{x}^{2} v\right)\right] .
\end{aligned}
$$

Using the estimates (30) and the fact that $\left\|G_{2 \nu t}\right\|_{L^{1}}=1$, we conclude that

$$
\int_{-\infty}^{\infty}\left|d_{1}(x, t)\right| d x \leq 16 \nu B^{2}
$$

Next we observe that

$$
\begin{aligned}
\int_{-\infty}^{\infty} \mid & d_{2}(x, t) \mid d x \\
\leq & 2\left\|\left(G_{2 \nu t} * v\right)\left(G_{2 \nu t} * \partial_{x} v\right)^{2}-\left(G_{2 \nu t} * \partial_{x} v\right)\left(G_{2 \nu t} * v \partial_{x} v\right)\right\|_{L^{1}} \\
& +2\left\|\left(G_{2 \nu t} * \partial_{x} v\right)\left(G_{2 \nu t} * v \partial_{x} v\right)-G_{2 \nu t} * v\left(\partial_{x} v\right)^{2}\right\|_{L^{1}}
\end{aligned}
$$

An application of Lemma 7(a) with $f=\partial_{x} v$ and $g=v$, together with the bounds given in expression (30), implies that

$$
2\left\|\left(G_{2 \nu t} * v\right)\left(G_{2 \nu t} * \partial_{x} v\right)^{2}-\left(G_{2 \nu t} * \partial_{x} v\right)\left(G_{2 \nu t} * v \partial_{x} v\right)\right\|_{L^{1}} \leq 16(\nu t)^{1 / 2} B^{3}
$$

Similarly, the equations (30) and Lemma $7(\mathrm{~b})$ with $f=v \partial_{x} v$ and $g=\partial_{x} v$ imply that

$$
2\left\|\left(G_{2 \nu t} * \partial_{x} v\right)\left(G_{2 \nu t} * v \partial_{x} v\right)-G_{2 \nu t} * v\left(\partial_{x} v\right)^{2}\right\|_{L^{1}} \leq 32(\nu t)^{1 / 2} B^{3} .
$$

Consequently,

$$
\int_{-\infty}^{\infty}\left|d_{2}(x, t)\right| d x \leq 48(\nu t)^{1 / 2} B^{3} .
$$

We conclude from Lemma $7(\mathrm{a})$ with $f=v \partial_{x}^{2} v$ and $g=v$ that

$$
\int_{-\infty}^{\infty}\left|d_{3}(x, t)\right| d x \leq 16(\nu t)^{1 / 2} B^{3} .
$$

The estimates for $d_{1}, d_{2}$ and $d_{3}$ imply that

$$
\int_{-\infty}^{\infty}|d(x, s)| d x \leq 16 \nu B^{2}+64(\nu t)^{1 / 2} B^{3},
$$

which by Lemma 6 implies that

$$
\begin{aligned}
\int_{-\infty}^{\infty}|b(x, t)| d x & \leq \int_{0}^{t} \int_{-\infty}^{\infty}|d(x, s)| d x d s \\
& \leq 16 \nu t B^{2}+43 \nu^{1 / 2} t^{3 / 2} B^{3} .
\end{aligned}
$$

If we substitute this result into expression (31) and recall that $k \leq 1 /(2 B)$, then we have that

$$
\|w(\cdot, k)\|_{L^{1}} \leq\left(8 \nu B^{2}+31 \nu^{1 / 2} B^{5 / 2}\right) k^{2} .
$$

This concludes the proof of the lemma.

Proof of the Viscous Splitting Theorem. The proof of the viscous splitting theorem can now be completed. Let $v_{j}=F_{k j} u_{0}$. The constant $B$ defined in Assumption 2 must bound $\left\|\partial_{x} v_{j}\right\|_{L^{\prime}}$ and all the spatial derivatives of $v_{j}$ up to second order. By Lemma 8, for time steps $k$ which satisfy Assumption $2(0 \leq k \leq 1 /(2 B))$,

$$
\left\|F_{k} v_{j}-\left[D_{k} A_{k}\right] v_{j}\right\|_{L^{\prime}} \leq C k^{2}
$$

where $C$ depends only on $u_{0}$ and $\nu$. 
To finalize the proof, we use an induction argument. For $0 \leq k \leq 1 /(2 B)$ let us make the induction assumption that

$$
\left\|F_{j k} u_{0}-\left[D_{k} A_{k}\right]^{j} u_{0}\right\|_{L^{1}} \leq C j k^{2} .
$$

Note that the initial induction hypothesis $(j=0)$ is obviously correct. Now the triangle inequality and the stability of the $A_{k}$ and $D_{k}$ operators, together with Eq. (32), imply that

$$
\begin{aligned}
& \left\|F_{(j+1) k} u_{0}-\left[D_{k} A_{k}\right]^{j+1} u_{0}\right\|_{L^{1}} \\
& \quad \leq\left\|F_{k} v_{j}-D_{k} A_{k} v_{j}\right\|_{L^{1}}+\left\|F_{j k} u_{0}-\left[D_{k} A_{k}\right]^{j} u_{0}\right\|_{L^{1}} \\
& \quad \leq C k^{2}+C j k^{2}=C(j+1) k^{2}
\end{aligned}
$$

which completes our proof of the viscous splitting theorem.

Acknowledgments. The author thanks Alexandre Chorin, Ole Hald and Gerry Puckett for helpful discussions. The work was supported in part by the Applied Mathematical Sciences subprogram of the Office of Energy Research, U. S. Department of Energy under contract DE-AC03-76SF00098.

Centre for Mathematical Analysis

Australian National University

GPO Box 4

Canberra ACT 2601, Australia

E-mail: SGR851@CSC.ANU.OZ.AU

1. J. T. BEALE \& A. MAJDA, "Rates of convergence for viscous splitting of the Navier-Stokes equation," Math. Comp., v. 37, 1981, pp. 243-259.

2. Y. BRENIER, "Averaged multivalued solutions for scalar conservation laws," SIAM J. Numer. Anal., v. 21, 1984, pp. 1013-1037.

3. Y. Brenier, A Particle Method for One Dimensional Non-Linear Reaction Advection Diffusion Equations, Comunicaciones Técnicas, Serie Naranja: Investigaciones, No. 351, 1983, Instituto de Investigaciones en Matematicas Aplicadas y en Sistemas, Universidad Nacional Autonoma de Mexico.

4. J. M. Burgers, "A mathematical model illustrating the theory of turbulence," Adv. in Appl. Mech., v. 1, 1948, pp. 171-199.

5. P. R. Chernoff, Product Formulas, Nonlinear Semigroups and Addition of Unbounded Operators, Mem. Amer. Math. Soc., No. 140, Amer. Math. Soc., Providence, R. I., 1974.

6. A. J. ChORIN, "Numerical study of slightly viscous flows," J. Fluid Mech., v. 57, 1973, pp. 785-796.

7. A. J. Chorin, "Vortex sheet approximation to boundary layers," J. Comput. Phys., v. 27, 1978, pp. 428-442.

8. A. J. Chorin, Numerical Methods for Use in Combustion Modelling, Proc. Internat. Conf. Numer. Methods in Science and Engineering, Versailles, 1979.

9. A. J. ChORIN \& J. MARSDEN, A Mathematical Introduction to Fluid Mechanics, SpringerVerlag, New York, 1979.

10. A. J. Chorin, T. J. R. Hughes, M. T. MCCracken \& J. E. Marsden, "Product formulas and numerical algorithms," Comm. Pure Appl. Math., v. 31, 1978, pp. 205-256.

11. K. L. Chung, A Course in Probability Theory, Academic Press, New York, 1974.

12. J. D. COLE, "On a quasi-linear parabolic equation occurring in aerodynamics," Quart. Appl. Math., v. 9, 1951, pp. 225236.

13. A. Eixstein, Investigation on the Theory of Brownian Movement, Translation, Methuen, London, 1956.

14. W. FELLER, An Introduction to Probability Theory and Its Applications, Vol. I, Wiley, New York, 1968 
15. W. Feller, An Introduction to Probability Theory and Its Applications, Vol. II, Wiley, New York, 1971.

16. A. Friedman, Partial Differential Equations of Parabolic Type, Prentice-Hall, N. J., 1964.

17. A. F. Ghoniem, A. J. Chorin \& A. K. Oppenheim, "Numerical modeling of turbulent flow in a combustion tunnel," Philos. Trans. Roy. Soc. London Ser. A, v. 304, 1982, pp. 303-325.

18. A. F. GHONIEM \& F. S. Sherman, "Grid-free simulation of diffusion using random walk methods," J. Comput. Phys., v. 61, 1985, pp. 1-37.

19. J. Goodman, "Convergence of the random vortex method," Comm. Pure Appl. Math., v. 40, 1987, pp. 189-220.

20. O. H. HALD, "Convergence of random methods for a reaction-diffusion equation," SIAM J. Sci. Statist. Comput., v. 2, 1981, pp. 85-94.

21. O. H. HALD, "Convergence of a random method with creation of vorticity," SIAM J. Sci. Statist. Comput., v. 7, 1986, pp. 1373-1386.

22. W. HoEfFDing, "Probability inequalities for sums of bounded random variables," J. Amer. Statist. Assoc., v. 58, 1963, pp. 13-30.

23. E. HOPF, "The partial differential equation $u_{t}+u u_{x}=\nu u_{x x}$," Comm. Pure Appl. Math., v. 3, 1950, pp. 201-230.

24. F. Jонn, Partial Differential Equations, 3rd ed., Springer-Verlag, New York, 1978.

25. S. N. KRUŽKOV, "First order quasilinear equations with several space variables," Math. USSR-Sb., v. 10, 1970, pp. 217-243.

26. J. A. Laitone, "A numerical solution for gas particle flows at high Reynolds numbers," $J$. Appl. Mech., v. 48, 1981, pp. 465-471.

27. C. MARChioro \& M. PUlvirenti, "Hydrodynamics in two dimensions and vortex theory," Comm. Math. Phys., v. 84, 1982, pp. 483-503.

28. F. J. MCGRATh, "Nonstationary plane flow of viscous and ideal fluids," Arch. Rational Mech. Anal., v. 27, 1968, pp. $329-348$.

29. A. K. Oppenheim \& A. Ghoniem, Application of the Random Element Method to One Dimensional Flame Propagation Problems, AIAA-83-0600, AIAA 21st Aerospace Sciences Meeting, Reno, Nevada, 1983.

30. R. D. Richtmyer \& K. W. MORTon, Difference Methods for Initial Value Problems, 2nd ed., Interscience, New York, 1967.

31. S. G. ROBERTS, Convergence of a Random Walk Method for the Burgers Equation, Ph.D. Thesis, University of California, Berkeley, 1985.

32. P. K. Stansby \& A. G. Dixon, "Simulation of flows around cylinders by a Lagrangian vortex scheme," Appl. Ocean. Res. Ser. 3, v. 5, 1984, pp. 167178

33. N. W. Sung, J. A. Laitone \& D. J. Pattinson, "Angled jet flow model for a diesel engine intake process random vortex method," Internat. J. Numer. Methods Fluids, v. 3, 1983, pp. 283293.

34. Z. TENG, "Elliptic-vortex method for incompressible flow at high Reynolds number," $J$. Comput. Phys., v. 46, 1982, pp. $54-68$.

35. J. J. W. VAN DER VEGT \& R. H. M. HUIJSMANS, "Numerical simulation of flow around bluff bodies at high Reynolds numbers," Z 50457, Netherlands Ship Model Basin, ONR Paper, August, 1982.

36. G. B. Whitна.M, Linear and Nonlinear Waves, Wiley-Interscience, New York, 1974. 